\title{
Some Finite Dimensional Integrable Systems and Their Scattering Behavior*
}

\author{
Mark Adler \\ Mathematics Research Center, University of Wisconsin, Madison, Wisconsin 53706, USA
}

\begin{abstract}
We consider a class of Hamiltonian systems which posses integrals expressible in terms of the eigenvalues of some associate matrices. Moreover, these systems will be solved explicitly and their scattering behavior investigated using additional associated matrices whose eigenvalues change in time during a flow.
\end{abstract}

\section{Introduction}

We consider Hamiltonian systems of $n$ particles on a line interacting with each other where the Hamiltonian is of the form:

$$
H_{\alpha}(x, y)=\frac{1}{2} \sum_{i=1}^{n} y_{i}^{2}+\sum_{1 \leqq i<j \leqq n} V\left(x_{i}-x_{j}\right)+\sum_{i=1}^{n} W\left(x_{i}\right) .
$$

The examples of such pairs of potentials $(V(x), W(x))$ to be considered are:

$$
\begin{aligned}
& \left(x^{-2},-\alpha^{2} x^{2} / 2\right), \\
& \left(\left(\frac{1}{2} \operatorname{coth} x / 2\right)^{2}, \alpha e^{x}\right) .
\end{aligned}
$$

Calogero and Marchioro [1] and Sutherland [2] have studied some of these potentials in the context of quantum mechanics, and their work suggested looking at the classical systems. For the case $\alpha=0$, Moser [3] has shown that both of the above examples are integrable systems, i.e., possess $n$ integrals whose associated Hamiltonian flows commute, and in addition the integrals are rational in $\left(x_{i}, y_{i}\right)$, $\left(e^{x_{i}}, y_{i}\right)$ respectively. The method he used was based on the isospectral technique of Lax [4], first applied by Flaschka [5] to the Toda lattice. This consists in the construction of a matrix function of $(x, y)$ whose spectrum remains fixed in $t$ if $x=x(t), y=y(t)$ are solutions of the above Hamiltonian system. We then take the

* Part of this research was done while at the Courant Institute of Mathematical Sciences, N.Y.U., with the Office of Naval Research, Contract no. N00014-76-C-03-01. In addition, it was sponsored by the United States Army under contract No. DAAG29-75-C-0024, and the National Science Foundation under Grant no. MCS75-17385 
eigenvalues of the matrix to be the desired integrals, and study systems whose Hamiltonians are functions of these integrals.

We extend this method to some new systems. Moreover, we construct a second matrix function of $(x, y, t)$ whose spectrum is invariant under the Hamiltonian flow, which allows us to describe the solutions more or less explicitly.

In this way we show for solutions $x=x(t)$ of the above Hamiltonian system, in Case (A), $\alpha \neq 0$, that the symmetric homogeneous polynomials in $x_{i}(t)$ of degree $v$ are polynomials of degree $v$ in $e^{ \pm \alpha t 1}$, which implies that all solutions of the system for $\alpha$ purely imaginary are periodic. Furthermore, for a class of systems whose Hamiltonians are integrals of the above system, the symmetric polynomials of $x_{i}(t)$ are polynomials of at most $n$ exponentials $e^{\lambda_{i} t}$. The same result is true for system (B), for $\alpha=0$, if we replace $x_{i}$ by $e^{x_{i}}$, as first proven by Olshanetzky and Perelomov ${ }^{2}$, and moreover for systems which we will construct such that their Hamiltonians are integrals of (B), $\alpha=0$. For (B), $\alpha \neq 0$, we find that the symmetric polynomials in $e^{x_{i}}$ are rational in $n$ exponentials $\mathrm{e}^{\lambda_{1} t}, \ldots, e^{\lambda_{n} t}$.

It is then easy to discuss the scattering behavior of the above systems quite explicitly in case the particles ultimately disperse, as in the case $\alpha>0$, and to construct scattering maps. For instance in Case (A), $\alpha>0$, the solutions behave asymptotically like:

$$
x_{k}(t)=\frac{1}{\sqrt{2}}\left\{q_{k}^{ \pm} e^{ \pm \alpha t}+\alpha^{-1} p_{k}^{ \pm} e^{\mp \alpha t}+O\left(e^{-2|\alpha t|}\right)\right\},
$$

for $t \rightarrow \pm \infty$, and for all $k, q_{k}^{ \pm}<q_{k+1}^{ \pm}$, if $x_{k}(0)<x_{k+1}(0)$. We include the artificial factors $2^{-1 / 2}, \alpha^{-1} 2^{-1 / 2}$, for a later purpose, to make $(x, y) \rightarrow(p, q)$ a canonical transformation. On the other hand for Case (B), $\alpha>0$, the solutions behave asymptotically like:

$$
x_{k}(t)= \pm \lambda_{k} t+\beta_{k}^{ \pm}+O\left(t^{-1}\right), \quad \text { for } t \rightarrow \pm \infty,
$$

where $\lambda_{1}<\lambda_{2}<\ldots<\lambda_{n}<0$, if $x_{i}(0)<x_{i+1}(0)$ for all $i$.

The scattering maps which we construct are canonical, given by polynomial relations, and lead to surprising algebraic transformations between the above systems (1.1). Moreover, they are found to agree with their own inverse, i.e., they are involutions. For instance in formula (1.3) we find

$$
\beta_{k}^{+}+\beta_{k}^{-}=2 \log \left(\alpha \lambda_{k}^{2}\right)
$$

It is surprising that the scattering map for the system (A), with $\alpha>0$, which relates data at $t=-\infty$ with data at $t=+\infty$ is precisely equal to the scattering map for the same system (A), with $\alpha=0$, but which relates data at $t=0$ with data at $t=\infty$.

1 Some of this work was announced and presented at the conference on Theory and Application of Solitons, held January 1976 in Tuscon, Arizona, and will appear in the proceedings of this conference [13]

2 I am indebted to F. Calogero for communicating to me the then unpublished results of Olshanetzky and Perelomov at the above mentioned conference. Their work, which was done independently has meanwhile been published in $[11,18]$. Part of their results overlap with some results presented in Sections 2-4 of this paper. The proofs presented are those of the unpublished preprint [12], which was handed out at the above mentioned conference of January 1976, in conjunction with a brief research announcement. The results of the preprint can be found in [13] 
Another interesting fact is that the scattering map for the system (A), with $\alpha>0$, which relates data at $t=0$ with data at $t=\infty$, transforms the Hamiltonian of that system into an integral of the system (B) with $\alpha=0$, after a trivial change of coordinates.

In Section 4 we show that Hamiltonian systems near by to system (1.1), Case (A) with $\alpha$ purely imaginary, have at least $n$ geometrically distinct orbits on each energy surface. While such results are easily derived in case the periodic solutions of the unperturbed system are isolated, they are quite delicate for manifolds of periodic orbits, which we encounter. This result follows directly from the perturbation theory of Weinstein [8].

\section{Integrability}

To construct the integrals of $H_{\alpha}(x, y)$ defined by (1.1), Case (A), we use the matrices of Moser's system, $Y=L(x, y), B=B(x)$, where $(x, y) \in \Omega=\left\{(x, y) \in R^{2 n} \mid x_{i}<x_{i+1}\right.$ for $i=1,2, \ldots, n-1\}, \Omega$ defined once and for all. We introduce

$$
\begin{aligned}
& Y_{j k}=[L(x, y)]_{j k}=\delta_{j k} y_{j}+i\left(1-\delta_{j k}\right)\left(x_{j}-x_{k}\right)^{-1} \\
& B_{j k}=i\left\{\delta_{j k}\left(-\sum_{s \neq j}\left(x_{j}-x_{s}\right)^{-2}+\left(1-\delta_{j k}\right)\left(x_{j}-x_{k}\right)^{-2}\right)\right\},
\end{aligned}
$$

and the diagonal matrices $X=\operatorname{diag}\left(x_{1}, x_{2}, \ldots, x_{n}\right), D(y)=\operatorname{diag}\left(y_{1}, y_{2}, \ldots, y_{n}\right)$. For future use, we observe the crucial fact that $L(x, y)$ has $n$ distinct real eigenvalues. (See [3].)

We observe the commutator relation $[X, Y]=C, C_{j k}=i\left(1-\delta_{j k}\right)$, noting that $i C$ is the identity operator on the subspace in $R^{n}$ specified by $\sum_{i=1}^{n} x_{i}=0$.

Define $D_{G}=\sum_{i=1}^{n}\left(G_{y_{i}} \partial_{x_{i}}-G_{x_{i}} \partial_{y_{i}}\right)$ to be the Hamiltonian vector field acting on functions of $(x, y)$, i.e. if $\dot{x}_{i}=G_{y_{i}}, \dot{y}_{i}=-G_{x_{i}}, i=1, \ldots, n$, then $d F(x, y) / d t=D_{G} F$. As an operator on matrices, $D_{G}$ acts componentwise. Let $\delta$ be the operator acting on matrix functions of $(x, y)$, and $t$, defined by

$$
\delta(Z)=\left(D_{H_{\alpha}} Z-[B, Z]\right)+\partial_{t} Z \text {. }
$$

Note that $\delta$ is a derivation, i.e., it satisfies the sum and product rule of differentiation, respecting order, for it is the sum of three derivations. We observe that in the case where $t$ doesn't occur explicitly in $Z$, then $\delta Z=D_{H_{\alpha}} Z-[B, Z]$. We also note for future reference, that I ited shall stand for the $n \times n$ identity matrix.

Theorem 1. If $x, y$ obey $\dot{x}_{i}=\partial H_{\alpha} / \partial y_{i}, \dot{y}_{i}=-\partial H_{\alpha} / \partial x_{i}, i=1, \ldots, n$, then the matrices $M^{ \pm}=(Y \pm \alpha X) e^{\mp \alpha t}$ satisfy the isospectral differential equation

$$
\delta M^{ \pm}=0,
$$

and consequently so does the time independent matrix

$$
E=\frac{1}{2} M^{+} M^{-}=\frac{1}{2}(Y+\alpha X)(Y-\alpha X) \text {. }
$$


Proof. We establish the following partial differential equation.
a) $\delta X=Y$,
b) $\delta Y=\alpha^{2} X$.

Note if we neglect the commutators, these equations become scalar harmonic oscillator equations. Since $\dot{x}=y$, we have $\dot{X}=D(y)$, and (2.2a) is just $D(y)=Y+[B, X]$, i.e., $Y=D(y)+[X, B]$, and since

$$
[X, B]_{j k}=\left(x_{j}-x_{k}\right) B_{j k}=i\left(1-\delta_{j k}\right)\left(x_{j}-x_{k}\right)^{-1}, \quad \text { where } P=-\frac{\alpha^{2}}{2} \operatorname{tr} X^{2},
$$

the definition of $Y$ yields the result. To prove (2.2b), we note

$$
H_{\alpha}=\frac{1}{2}\left[\operatorname{tr} Y^{2}-\alpha^{2} \operatorname{tr} X^{2}\right]=H_{0}+P,
$$

and so $D_{H_{\alpha}} Y=D_{H_{0}} Y+D_{P} Y$, but in [3] it is computed that $D_{H_{0}} Y=[B, Y]$ (a straightforward computation), and since

$$
D_{P} Y=D_{P}(D(y))=\alpha^{2} X,
$$

we have

$$
D_{H_{\alpha}} Y=D_{H_{0}} Y+D_{P} Y=[B, Y]+\alpha^{2} X,
$$

and (2.2b) is proven. Now (2.2) immediately implies

$$
\delta(Y \pm \alpha X)= \pm \alpha(Y \pm \alpha X)
$$

hence

$$
\begin{aligned}
\delta M^{ \pm} & =(\delta(Y \pm \alpha X)) e^{\mp \alpha t}+(Y \pm \alpha X)\left(\delta\left(e^{\mp \alpha t} I\right)\right) \\
& = \pm \alpha(Y \pm \alpha X) e^{\mp \alpha t}+(Y \pm \alpha X)\left(\mp \alpha e^{\mp \alpha t}\right)=0
\end{aligned}
$$

and thus

$$
\delta M^{ \pm}=0 .
$$

That $E$ satisfies $\delta E=0$ is an immediate consequence of $\delta$ being a derivation.

Remark *1. We note that the operator equation $\delta D=0$, or equivalently $U^{-1}(\delta D) U=0=(d / d t)\left(U^{-1} D U\right)$, where $\dot{U}=B U$; this is equivalent to $U(t)^{-1} D(t) U(t)=U^{-1}(0) D(0) U(0)$, provided $U(t), U(t)^{-1}$ exists. We say $D$ undergoes an isospectral deformation in time. Thus the constants of the derivation $\delta$, i.e., the algebra which forms the kernel of the operator $\delta$, are nothing but the isospectral matrices which evolve through their similarity class via the infinitesimal generator $B$. Their eigenvalues and all functions of them remain constant in time.

Remark 2. In our case $B+B^{*}=0$, and $U(t)$ exists for all finite time, and $U U^{*}=I$ if it holds at some time $t_{0}$. If in addition $U(t)$ converges for $t \rightarrow \infty$, we may set the value of $U$ at $t=\infty$ to be $I$, the $n \times n$ identity matrix. Thus $U(t)=I-\int_{t}^{\infty} B(x(s)) U(s) d s$, and we now redefine $U(x, y)$ as the solution of the integral equation $U(x, y)=I-\int_{0}^{\infty} B(x(s)) U(x(s), y(s)) d s$, as a function of $x=x(0), y=y(0)$ on $\Omega$, 
rather than by $U_{1 t=0}=I$. In other words we construct $U$ via the above integral equation, thus directly exhibiting its dependence on $\Omega$. Then $U(x, y)$ is a solution of the partial differential equation $D_{H_{\alpha}} U=B U$.

Thus for every point $(x, y)$ of $\Omega$ we have $U(x, y), B(x)$, and that $U^{-1}(x, y) Y(x, y) U(x, y)$ is constant along orbits and equals $\lim _{t \rightarrow \infty} Y(x(t), y(t))$. If, as in Case (A), $\alpha=0, \lim _{t \rightarrow \infty} Y(x(t), y(t))$ is a diagonal matrix, then the columns of $U(x, y)$ are the eigenvectors of $Y(x, y)$.

If in some way we can find a normalization of the eigenvectors, and hence of $U(x, y)$, we may dispense with its definition using the partial differential equation in $(x, y)$ space. For instance, if $B$ satisfies an equation of the form $T B=0$, with $T$ a constant matrix with no column identically zero, then automatically $T U=T$ is the desired normalization. For $d T U / d t=D_{H}(T U)=T B U=0$, but $\lim _{t \rightarrow \infty} U(x(t), y(t))=I$ by definition, and $T I=T$, hence $T U(x, y)=T$ for all $(x, y)$, i.e., $U(x, y)$ is a member of the Lie subgroup of the unitary group specified by $T U=T$. This symmetry coupled with the fact that $U$ diagonalizes $L$, algebraically defines the matrix function $U$ if the eigenvalues are distinct. The above happy situation of simple eigenvalues occurs for all cases we will consider, and $T$ will be of the form $T_{k j}=i$ for all $k, j$, and $U$ is algebraically defined by $U^{-1} Y U=$ diagonal matrix, and $T U=T$.

Remark 3. We note that $E$ does not contain time explicitly and so $I_{j}(E)=\operatorname{tr} E^{j}$, $j=1, \ldots, n$ are $n$ rational integrals of the motion.

Letting $z=(x, y)$, we define

$$
\{f, g\}_{z}=\left\langle J \nabla_{z} f, \nabla_{z} g\right\rangle=\sum_{j=1}^{n} \partial(f, g) / \partial\left(x_{i}, y_{i}\right),
$$

i.e., the Poisson bracket of $f, g$, computed in $z=(x, y)$ coordinates, where $\langle$,$\rangle is the$ ordinary scalar dot product in $R^{2 n}, \nabla_{z}$ the gradient operator in $R^{2 n}$ equipped with the dot product $\langle$,$\rangle , and J$ the $2 n \times 2 n$ constant matrix $\left[\begin{array}{cc}0, & -I \\ I, & 0\end{array}\right]$, with $I$ the $n \times n$ identity matrix. Note $D_{g} f=\{f, g\}$ by the previous definition of $D_{g}$.

Theorem 2. The $I_{j}(E)$ are in involution, i.e., $\left\{I_{j}, I_{k}\right\}=0$ for $j, k=1,2, \ldots, n$. The $I_{j}$ are algebraically independent, i.e., the system $H_{\alpha}$ in Case (A) is classically integrable.

To prove the theorem, we use the asymptotic description $(\alpha>0)$ of the orbits. Clearly it suffices to prove that $\phi_{j k}=\left\{I_{j}, I_{k}\right\}$ vanishes in some neighborhood, since $\phi_{j k}(x, y)$ are rational functions of their arguments. Also it suffices to consider the case of $\alpha>0$, as $\alpha$ enters rationally into $\phi_{j k}$. The algebraic independence of the $I_{j}(x, y)$ is easily seen upon letting $x_{i+1}-x_{i}$ be large for all $i$. For $\alpha>0$ we use the asymptotic behavior of the solution, letting $\alpha=1$ for simplicity.

Lemma 2.1. For $\left(q_{1}<q_{2}<\ldots<q_{n}\right),\left(p_{1}, p_{2}, \ldots, p_{n}\right)$ varying in some appropriate neighborhood $N$ of $R^{2 n}$, there exists solutions of our system for $t \geqq 0$, satisfying:

$$
\left\{\begin{array}{l}
x_{j}(t, p, q)=2^{-\frac{1}{2}}\left(q_{j} e^{t}+p_{j} e^{-t}\right)+O\left(e^{-\gamma t}\right) \\
y_{j}(t, p, q)=2^{-\frac{1}{2}}\left(q_{j} e^{t}-p_{j} e^{-t}\right)+O\left(e^{-\gamma t}\right)
\end{array}\right\} \text { for } t \rightarrow+\infty
$$


where $\gamma$ is any number such that $2<\gamma<3$, and where the above expression may be differentiated with respect to $(p, q)$ and still remains valid. Moreover, the map: $(p, q) \rightarrow(x(0), y(0))$ is canonical.

We sketch the proof. For $u_{j}(t, p, q)=x_{j}(t, p, q)-\frac{1}{2^{-1 / 2}}\left(q_{j} e^{t}+p_{j} e^{-t}\right)$, we have the following integral equation

$$
u_{j}=\int_{t}^{\infty} k(s-t) V_{j}[u, s] d s=K[u]
$$

where $k(t)=\sinh t$,

$$
\begin{aligned}
& V_{j}[u, s]=-\left(\partial / \partial x_{j}\right) H_{0}\left[u(s, p, q)+2^{-\frac{1}{2}}\left(q e^{t}+p e^{-t}\right)\right], \\
& u(t, p, q): R^{+} \times N=W \rightarrow R^{n},
\end{aligned}
$$

where $R^{+}=\{s \mid s \geqq 0\}$, and $N$ is the neighborhood in $R^{2 n}$ to be determined. For $\varepsilon>0$, we apply the contraction principle to the set $\mathscr{B}=\mathscr{B}(\varepsilon)$ of vector $C^{2}$-functions $u=u(t, p, q)$ of $W$, with the restriction that $\sup _{W}(|u|+|\partial u / \partial t|) e^{\gamma t}=\|u\| \leqq \varepsilon$. We then pick $M_{\varepsilon}$ sufficiently large depending on $\varepsilon$, fix a $q^{0}$ having the property $\min _{i<j}\left|q_{i}^{0}-q_{j}^{0}\right|>M_{\varepsilon}, q_{i}^{0}<q_{i+1}^{0}$, and define $N=\left\{(q, p)|| p|+| q-q^{0} \mid<M_{\varepsilon}^{-1}\right\}$. Then one verifies in a standard manner that $K[B] \subset[B]$, and $\left\|K\left[u_{1}\right]-K\left[u_{2}\right]\right\|<1 / 2\left\|u_{1}-u_{2}\right\|$ for $u_{1}, u_{2} \in \mathscr{B}$. Hence via the contraction principle, $u=K[u]$ possesses a unique solution $u_{0}$. If we define the vector $v=(u, \partial u / \partial q, \partial u / \partial p)$, $\|v\|=\sup _{W}(|v|+|\partial v / \partial t|) e^{\gamma t}$, then a similar argument with this new norm will yield the existence of $\partial u / \partial q, \partial u / \partial p$, their derivatives with respect to time, and the asymptotic estimates on these quantities stated in (2.3).

Thus a solution to (2.3) has been constructed, and only the last statement of the Lemma 2.1 remains to be proven. We now have the map $\tau_{t}$, where

$$
\tau_{t}:\left(\begin{array}{l}
q \\
p
\end{array}\right) \rightarrow\left(\begin{array}{l}
x(t, q, p) \\
y(t, q, p)
\end{array}\right), \quad t \geqq 0, \quad(q, p) \in N .
$$

By the statement of the first part of the lemma, for $t$ sufficiently large, $\tau_{t}$ is $C^{\prime}$ invertible, on perhaps a smaller neighborhood, and

$$
\frac{\partial(x(t, q, p), y(t, q, p))}{\partial(q, p)}=\frac{1}{\sqrt{2}}\left[\begin{array}{rr}
I, & I \\
I, & -1
\end{array}\right]+O\left(e^{-\gamma t}\right) .
$$

Now the map $\phi^{t}:(x(0), y(0)) \rightarrow(x(t), y(t))$ is canonical and hence $C^{\prime}$ invertible, thus $\tau_{0}=\phi^{-t} \tau_{t}$ is $C^{\prime}$ invertible in $N$. If we denote the Jacobian matrix of these maps by $j$, we have

a) $j\left(\tau_{0}^{-1}\right)=j\left(\tau_{t}^{-1}\right) \cdot j\left(\phi^{t}\right)$

b) $j^{T}\left(\tau_{t}\right) J j\left(\tau_{t}\right)=-J+O\left(e^{-\gamma t}\right)$, as a consequence of (2.5),

c) $j^{T}\left(\phi^{t}\right) J j\left(\phi^{t}\right)=J$, since $\phi^{t}$ is a canonical map,

d) $\left\|j\left(\phi^{t}\right)\right\| \leqq C e^{|t|}$.

As a consequence of a)-d) we conclude $j\left(\tau_{0}^{-1}\right)^{T} J j\left(\tau_{0}^{-1}\right)=-J+O\left(e^{-(\gamma-1)|t|}\right)$ for all $t$. Hence $j\left(\tau_{0}^{-1}\right)^{T} J j\left(\tau_{0}^{-1}\right)=-J$, and thus $\tau=\tau_{0}$ is canonical, and the lemma is proven. We observe a map preserves the form of the previously defined Poisson 
bracket if and only if its Jacobian at every point belongs to the symplectic group $\operatorname{Sp}(2 n, R)$ of $2 n \times 2 n$ matrices satisfying $A^{T} J A=J$, which is easily verified using the definition of $\{$,$\} . More generally we consider a transformation whose Jacobian A$ satisfies $A^{T} J A=\mu J$, with a constant $\mu \neq 0$ and call it a canonical transformation with multiplier $\mu$.

Proof of Theorem. So far we have proven the map $(x(0), y(0)) \rightarrow(q, p)$ is canonical, but with multiplier -1 , i.e. $\{,\}_{z}=-\{,\}_{r}, r=(q, p)$. We define the map $\psi:(q, p) \rightarrow(\xi, \eta)$ by

$$
\xi_{i}=\log q_{i}, \quad \eta_{i}=-q_{i} p_{i}
$$

in the domain $0<q_{1}<\ldots<q_{n}$. This map is clearly canonical with multiplier -1 , and hence $\psi \circ \tau^{-1}: \tau(N) \rightarrow \psi(N)$ is canonical with multiplier 1 .

Now we recall that $(\alpha=1$, for simplicity),

$$
E=\frac{1}{2} M^{+} M^{-}=\frac{1}{2}(Y+X)(Y-X)=\frac{1}{2}\left(Y^{2}-X^{2}\right)+\frac{1}{2}[X, Y],
$$

hence

$$
\begin{aligned}
2 E_{k l}= & \delta_{k l}\left(y_{k}^{2}-x_{k}^{2}+\sum_{m}^{\prime}\left(x_{k}-x_{m}\right)^{-2}\right) \\
& +\left(1-\delta_{k l}\right)\left\{\frac{i\left(y_{k}+y_{l}\right)}{\left(x_{k}-x_{k}\right)}+i+\sum_{m}^{\prime}\left(x_{k}-x_{m}\right)^{-1}\left(x_{l}-x_{m}\right)^{-1}\right\},
\end{aligned}
$$

where the prime indicates that undefined terms are to be left out. Using (2.3), (2.6), we conclude

$$
\left\{\begin{aligned}
\lim _{t \rightarrow \infty} E(x(t), y(t))= & E(\infty)=\left\{\delta_{k l} \eta_{k}+\frac{1}{2} i\left(1-\delta_{k l}\right) \operatorname{coth} \frac{1}{2}\left(\xi_{k}-\xi_{l}\right)\right. \\
& \left.+\frac{1}{2} i\left(1-\delta_{k l}\right)\right\} \\
= & Z(\eta, \xi)+\frac{1}{2} C=Z^{+}(\xi, \eta), \quad C_{k l}=i\left(1-\delta_{k l}\right)
\end{aligned}\right\},
$$

where $Z(\xi, \eta), Z^{+}(\xi, \eta)$, are defined by this relation, and we define $Z^{-}(\eta, \xi)$ $=Z(\xi, \eta)-\frac{1}{2} C$. Now if

$$
F(\xi, \eta)=\frac{1}{2} \operatorname{tr} Z^{2}=\frac{1}{2} \sum_{i=1}^{n} \eta_{i}^{2}+\frac{1}{4} \sum_{i<j} \operatorname{coth}^{2} \frac{\left(\xi_{i}-\xi_{j}\right)}{2},
$$

i. e., $F(\xi, \eta)=H_{\alpha}(\xi, \eta)$ of (1.1) Case (B), $\alpha=0$, then the flow defined by

$$
d \xi_{i} / d s=\partial F / \partial \eta_{i}=\eta_{i}, \quad d \eta_{i} / d s=-\partial F / \partial \xi_{i}, \quad i=1, \ldots, n,
$$

can also be expressed by an isospectral deformation of the form $\dot{Z}=D_{F} Z=[K, Z]$, see [3]. Here $K$ has off diagonal elements

$$
K_{k l}(\xi)=i\left[\frac{1}{2} \sinh ^{-1}\left(\xi_{k}-\xi_{l}\right)\right]^{2}=i q_{k} q_{l}\left(q_{k}-q_{l}\right)^{-2},
$$

and the sum of the rows, (and columns) is zero, as was the case for $B$. Hence $C K=-i K=K C$ implies $[C, K]=0=D_{F} C$, and therefore $Z^{+}(\xi, \eta)=Z(\xi, \eta)+\frac{1}{2} C$, [and similarly $Z^{-}(\xi, \eta)=Z(\xi, \eta)-\frac{1}{2} C$ ], satisfies the same isospectral differential equation as $Z$. In addition, under the flow induced by $F$, we find

$$
\begin{aligned}
& \xi_{i}=\alpha_{i} s+\beta_{i}+O\left(s^{-1}\right), \quad \text { for } \quad s \rightarrow+\infty \\
& \eta_{i}=\alpha_{i}+O\left(s^{-2}\right),
\end{aligned}
$$


with $\alpha_{i}<\alpha_{i+1}$, and in fact the transformation $(\xi, \eta) \rightarrow(\beta, \alpha)$ is canonical by a similar argument as in Lemma 2.1, see [3] for details. However, we will sketch a proof that the $\alpha_{i}^{\prime}$ 's are in involution. By (2.7), (2.10), the distinct $\alpha_{i}$ 's constitute the simple spectrum of the matrix $Z^{+}(\xi, \eta)$ [and also $Z^{-}(\xi, \eta)$ ], which undergoes an isospectral deformation. Hence $\left\{\alpha_{i}, \alpha_{j}\right\}_{\zeta}, \zeta=(\xi, \eta)$, is a constant of the motion, which we evaluate at $s=\infty$. But since $\eta_{i} \rightarrow \alpha_{i}$, for $s \rightarrow \infty$, in the $C^{1}$ sense, $0=\left\{\eta_{i}, \eta_{j}\right\}_{\zeta} \rightarrow\left\{\alpha_{i}, \alpha_{j}\right\}_{\zeta}$, and thus $0=\left\{\alpha_{i}, \alpha_{j}\right\}_{\zeta}$. Now since the matrices $E(x, y)$ and $\lim _{t \rightarrow \infty} E(x(t), y(t))$ $=Z^{+}(\xi, \eta)$ and $\lim _{s \rightarrow \infty} Z^{+}(\xi(s), \eta(s))$ are unitarily equivalent via Remark 1 , while all the maps considered are canonical, we conclude that the eigenvalues of $E(x, y)$, which are the $\alpha_{1}, \ldots, \alpha_{n}$, are in involution, and hence so are the $I_{j}(E)=\sum_{k=1} \alpha_{k}^{j}$, being functions of the eigenvalues. Thus the proof of Theorem 2 is completed.

\section{Scattering Theory}

To discuss the scattering theory of the system of Section 2, we need a lemma, which will be a consequence of the following:

Theorem 3. The solutions of the system $\dot{x}_{i}=\partial H_{\alpha} / \partial y_{i}, \dot{y}_{i}=-\partial H_{\alpha} / \partial x_{i}, i=1, \ldots, n$, obey

$$
\sum_{i=1}^{n} x_{i}^{v}(t)=\sum_{s=0}^{v} c_{s}^{(v)} e^{(2 s-v) \alpha t}
$$

$v$ a natural number, $c_{s}^{(v)}$ a rational function of initial data. Hence the $x_{i}(t)$ are explicitly given as algebraic functions of $e^{ \pm \alpha t}$, and initial data $(\alpha \neq 0)$.

Proof. Referring back to the definitions of Section 2, one computes

$$
X=\frac{1}{2} \alpha^{-1}\left(M^{+} e^{\alpha t}-M^{-} e^{-\alpha t}\right),
$$

since $M^{ \pm}=(Y \pm \alpha X) e^{\mp \alpha t}$.

Raising both sides of Equation (3.2) to the $v^{\text {th }}$ power, and then taking the trace, we conclude

$$
\sum_{i=1}^{n} x_{i}^{v}=\sum_{s=0}^{v} c_{s}^{(v)} e^{(2 s-v) \alpha t},
$$

where $c_{s}^{(v)}=\operatorname{tr} C_{s}^{v}$, and $C_{s}^{(v)}$ is a sum containing all possible $v$-fold product of the noncommuting matrices $M^{+}, M^{-}$taken $s$, and $v-s$ times respectively. We recall the algebra generated by $M^{+}, M^{-}$undergoes an isospectral deformation during the flow, by Remark 1 of Section 2. Thus $C_{s}^{(v)}$ undergoes an isospectral deformation during the flow, and $\operatorname{soc}_{s}^{(v)}(t)=c_{s}^{(v)}(0)$ is an integral which is a rational function of the initial data. We amplify Lemma 2.1 , and now prove a statement about the scattering of all solutions. Since the particles can't collide we may order them so that $x_{i}<x_{i+1}$ for $i=1,2, \ldots, n-1$, and all real $t$.

Lemma 3.1. For $\alpha>0$, every solution satisfies

$$
x_{k}(t)=2^{-1 / 2}\left(q_{k} e^{\alpha t}+\alpha^{-1} p_{k} e^{-\alpha t}\right)+O\left(e^{-3 \alpha t}\right),
$$


as $t \rightarrow+\infty, q_{j}<q_{j+1}, j=1,2, \ldots, n-1$, under the hypothesis of Theorem 3 , with a similar conclusion for $t \rightarrow-\infty$.

Proof. We note in Theorem 3 that $c_{v}^{(v)}=\operatorname{tr}\left[\frac{\alpha^{-1}}{2}\left(Y_{0}+\alpha X_{0}\right)\right]^{v}$, where the subscript indicates evaluation at $t=0$. Since $Y \pm \alpha X=L(x, y \pm \alpha x)$, we conclude $Y \pm \alpha X$ has simple spectrum, for we observed at the beginning of Section 2 that matrices of the form $L(x, y)$ have simple spectrum. Multiplying (2.1) by $e^{-\alpha v t}$, we find

$$
\sum_{j}\left(x_{j} e^{-\alpha t}\right)^{v}=c_{v}^{(v)}+c_{v-1}^{v} e^{-2 \alpha t}+O\left(e^{-4 \alpha t}\right),
$$

with $c_{v}^{(v)}=\left(2^{-v / 2} \sum_{j} q_{j}^{v}\right.$, where $q_{1}<q_{2}<\ldots<q_{n}$ are the distinct time-independent eigenvalues of $2^{-1 / 2} \alpha^{-1} M^{+}$. Since $x_{1}, x_{2}, \ldots, x_{n}$ are distinct and ordered, they are uniquely determined by $\sum_{j=1}^{n} x_{j}^{v}$, and hence by the expression for $c_{v}^{(v)},(3.4)$, and by the distinctness of the $x_{i}$ 's and $q_{i}$ 's respectively, we have

$$
x_{i} e^{-\alpha t}=2^{-1 / 2}\left(q_{i}+\alpha^{-1} p_{i} e^{-2 \alpha t}\right)+O\left(e^{-4 \alpha t}\right) .
$$

This defines the $p_{i}$. Upon multiplication of both sides of the latter expression by $e^{\alpha t}$, the lemma follows.

Remark 1. We thus have a map $\phi_{+}: \Omega \rightarrow \Omega, \Omega$ defined in the beginning of Section 2 , given by $\phi_{+}(x, y)=(q, p)$ for $\alpha$ real. We wish to show it is a bijection of $\Omega$. Given $q_{i}<q_{i+1}, p_{i}$, we may form

$$
x_{i}(t)=2^{-1 / 2}\left(q_{i} e^{\alpha t}+\alpha^{-1} p_{i} e^{-\alpha t}+\int_{t}^{\infty} \sinh (s-t) V_{i}[x(s)] d s, \quad i=1,2, \ldots, n,\right.
$$

and prove the existence of a solution $x(t)$, for $t \geqq t_{0}$, with the given $(q, p)$, by the methods of Lemma 2.1. From the conservation of the energy, $H_{\alpha}(x, y)$, and the behavior in time of $\sum_{i=1}^{n} x_{i}^{2}$ given by (3.1), we conclude $\sum_{i=1}^{n}\left(\left|x_{i}\right|+\left|y_{i}\right|\right) \leqq C e^{|\alpha t|}$, and therefore $x(t)$ exists and is unique for all time, and in particular at $t=0$. Whereas Lemma 3.1 enables us to define $\phi_{+}$on $\Omega$, the above discussion shows that $\phi_{+}^{-1}$ is well-defined on $\Omega$, and hence $\phi_{+}$is a bijection of $\Omega$, and canonical, by the arguments of Lemma 2.1.

We now exhibit an important feature of $\phi_{+}$, namely that it is an algebraic mapping, i.e., it is implicitly given by polynomial relations, and it is an involution, i.e., $\phi_{+}{ }^{\circ} \phi_{+}=$identity. Then we shall discuss a surprising relation of $\phi_{+}$with the scattering map in the case $\alpha=0$, and rational invariants of this map.

The algebraic nature of the map is given by matrix equivalences. Referring to Remark 2 in Section 2, since $|B|=O\left(t^{-2}\right)$ along an orbit, $B$ being the infinitesimal generator of the isospectral flow, we may define the function $U(x, y)$, and by $(3.5)^{+}$ and the same remark, in a purely algebraic manner, and thus conclude $\lim _{t \rightarrow \infty} M^{ \pm}(t)$ exists and equals $U^{-1}(x, y) M^{ \pm}(x, y) U(x, y)$. Since

$$
\begin{aligned}
M^{ \pm} & =(Y \pm \alpha X) e^{\mp \alpha t}, \\
{\left[M^{ \pm}(z(t), t)\right]_{k j} } & =\delta_{k j}\left(y_{j}(t) \pm \alpha x_{j}(t)\right) e^{\mp \alpha t}+i\left(1-\delta_{j k}\right)\left(x_{k}(t)-x_{j}(t)\right)^{-1} e^{\mp \alpha t},
\end{aligned}
$$


and upon using $y_{i}(t)=\dot{x}_{i}(t),(3.3)$, we find for $t \rightarrow \infty$,

$$
\left\{\begin{array}{ll}
\left(y_{i}+\alpha x_{i}\right)(t) e^{-\alpha t} \rightarrow 2^{1 / 2} \alpha q_{i}, & \left(y_{i}-\alpha x_{i}\right)(t) e^{\alpha t} \rightarrow-2^{1 / 2} p_{i}, \\
e^{-\alpha t}\left(x_{i}(t)-x_{j}(t)\right)^{-1} \rightarrow 0, & \left.e^{\alpha t}\left(x_{i}-x_{j}\right)(t) \rightarrow 2^{1 / 2}\left(q_{i}-q_{j}\right)^{-1}\right)
\end{array}\right\},
$$

and so we have (see Remark 2 of Section 2),

$$
\left\{\begin{array}{ll}
(+) & U^{-1}(x, y) M^{+}(x, y) U(x, y)=\lim _{t \rightarrow \infty} M^{+}(z(t), t)=2^{1 / 2} \alpha Q \\
(-) & U^{-1}(x, y) M^{-}(x, y) U(x, y)=\lim _{t \rightarrow \infty} M^{-}(z(t), t)=-2^{1 / 2} P^{T}
\end{array}\right\},
$$

where $Q=\operatorname{Diag}\left(q_{1}, \ldots, q_{n}\right), P=L(q, p)$.

It is important to notice that both matrices $M^{ \pm}(x, y)$ are transformed in (3.5) by the same similarity transformation. Now let $L_{\alpha}(x, y)=2^{-1 / 2} \alpha^{-1} L^{T}(x, y)$ $=2^{-1 / 2} \alpha^{-1} L(-x, y)$, and let $\sim$ denote spectral equivalence, remembering $X=\operatorname{diag}\left(x_{1}, \ldots, x_{n}\right)$, we obtain :

Theorem 4. The scattering map $\phi_{+}$is given by the following symmetric relations

$$
\begin{aligned}
& X \sim L_{\alpha}(q, p+\alpha q), \\
& L_{\alpha}^{T}(x, y+\alpha x) \sim Q,
\end{aligned}
$$

were both equivalences are effected by the same similarity transformation $U^{T}(x, y)$. From the symmetry of (3.6) we see at once $\phi_{+}^{-1}=\phi_{+}$, i.e., $\phi_{+}{ }^{\circ} \phi_{+}=\mathrm{id}$.

Proof. Line (3.6b) is the transpose of Equation (3.5) ${ }^{+}$, while (3.6a) is the transpose of the difference of Equations (3.5) ${ }^{ \pm}$, thus finishing the proof.

We now study the scattering map $\phi$ from $t \rightarrow-\infty$ to $t \rightarrow+\infty, \phi=\phi_{+}{ }^{\circ}\left(\phi_{-}\right)^{-1}$, where $\phi_{-}$denotes the map from the initial data $(x(0), y(0))$ into the scattering data $\left(q^{-}, p^{-}\right)$for $t=-\infty$, defined by $x_{i}=2^{-1 / 2}\left(q_{i}^{-} e^{-\alpha t}+\alpha^{-1} p_{i}^{-} e^{\alpha t}\right)+O\left(e^{2 \alpha t}\right), t \rightarrow-\infty$. To compute $\phi$, we may restrict ourselves to the case $\alpha=1$. We recall that $L_{1}(x, y)$ $=2^{-1 / 2} L^{T}(x, y)$, and we conlcude from Theorem 4 , that $\phi_{+}, \phi_{-}$are given implicitly by:

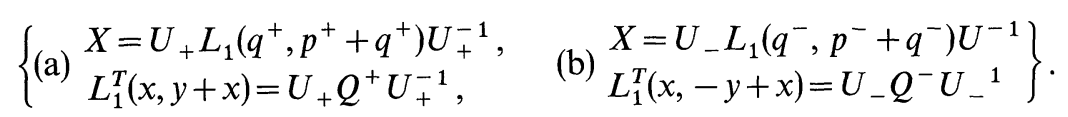

We may derive (b) from (a) by using the time reversibility of the differential equations under $(t, x, y) \rightarrow(-t, x,-y)$, hence $\phi_{-}=\phi_{+}{ }^{\circ} \varrho$, where $\varrho(x, y)=(x,-y)$.

Therefore we conclude from (3.7),

$$
\begin{aligned}
U_{-} Q^{-} U_{-}^{-1} & =L_{1}^{T}(x,-y+x)=L_{1}^{T}(x,-(y+x))+2^{1 / 2} X \\
& =-L_{1}(x, y+x)+2^{1 / 2} X^{T} \\
& =\left(U_{+}^{T}\right)^{-1}\left\{-Q^{+T}+2^{-1 / 2} L_{1}^{T}\left(q^{+}, p^{+}+q^{+}\right)\right\} U_{+}^{T} \\
& =\left(U_{+}^{T}\right)^{-1}\left\{2^{-1 / 2} L_{1}^{T}\left(q^{+}, p^{+}\right)\right\} U_{+}^{T},
\end{aligned}
$$

and after a similar calculation involving $Q^{+}$we conclude:

Theorem 5. For Case (A), $\alpha>0$, the scattering map

$$
\phi=\phi_{+}{ }^{\circ} \phi_{-}^{-1}
$$


is given implicitly by

$$
Q^{-} \sim L^{T}\left(q^{+}, p^{+}\right), \quad L\left(q^{-}, p^{-}\right) \sim Q^{+},
$$

where both of the equivalences are brought about by the same unitary map, $V=U_{+}^{T} U_{-}$.

The case $\alpha=0$ has been treated by Moser, but for completeness we include short proofs in the spirit of the above arguments, and then relate the results of the two cases. It was shown in [3], that if $x$, obey $\dot{x}_{i}=\partial H_{0} / \partial y_{i}, \dot{y}_{i}=-\partial H_{0} / \partial x_{i}, i=1, \ldots, n$, and if $x_{i}(0)<x_{i+1}(0), i=1, \ldots, n-1$, then as $t \rightarrow \infty$

$$
\begin{aligned}
x_{i} & =q_{i} t+p_{i}+O\left(t^{-1}\right), \quad y_{i} \rightarrow q_{i}+O\left(t^{-2}\right), \quad q_{i}<q_{i+1}, \\
i & =1,2, \ldots, n-1 .
\end{aligned}
$$

In fact, if we define the map $\psi_{+}$by $\psi_{+}(x, y)=(q, p), \psi_{+}: \Omega \rightarrow \Omega$ where $\Omega$ is the domain used in the definition of $\phi_{+}$, then $\psi_{+}$is seen to be a canonical bijection by arguments similar to ones given here. We are now in a position to state:

Theorem 6. $\psi_{+}=\phi$, where $\phi=\phi_{+}{ }^{\circ} \phi_{-}^{-1}$.

Moreover $\phi=\phi_{+}{ }^{\circ} \phi_{-}^{-1}=\phi_{+}{ }^{\circ} \varrho^{\circ} \phi_{+}^{-1}$, so $\psi_{+}$is "conjugate" to the linear reflection $\varrho$, and hence is an involution.

The equation $\psi_{+}=\phi$ expresses that the scattering map $\phi$ relating data at $t=-\infty$ to data at $t=+\infty$ for the system $H_{\alpha}$, is precisely the scattering map $\psi_{+}$ relating data at $t=0$ to data at $t=\infty$ for the system $H_{0}$. Even the unitary maps in the two cases, which effect the matrix transformations are the same, as they both are contained in the Lie subgroup discussed in Remark 2 of Section 2.

Proof. We now observe that

$$
M^{+}=M^{+}(\alpha), \quad M(\alpha)=\frac{1}{2}\left(M^{+}-M^{-}\right)=X \cosh \alpha t-\alpha^{-1} \sinh \alpha t Y,
$$

are isospectral matrices under the flow $H_{\alpha}$ (see Remark 1 of Section 2), and for $\alpha \rightarrow 0$ we obtain $L(x, y), X-t L(x, y)$ as isospectral matrices under the flow induced by $H_{0}$. Using (3.8), and the arguments of the Lax formalism used previously, we conclude:

$$
\left\{\begin{array}{l}
Q=\lim _{t \rightarrow \infty} L(x, y) \sim L(x(0), y(0)) \\
L^{T}(q, p)=L(-q, p)=\lim _{t \rightarrow \infty}(X-t L(x, y)) \sim X(0)
\end{array}\right\},
$$

where $\sim$ denotes unitary equivalence.

Hence $\psi_{+}(x(0), y(0))=(q, p)$ is explicitly given by

$$
X(0) \sim L^{T}(q, p), \quad L(x(0), y(0)) \sim Q,
$$

where both equivalences are effected by the same similarity transformation. This completes the proof of the theorem, by comparing this with Theorem 5 .

The map $\psi_{+}$linearizes the flow for the case $\alpha=0$, and is a algebraic, yet we have only an implicit description of it. However, we can find some rational functions invariant under the action of $\psi_{+}$, similarly for $\phi_{+}$.

Theorem 7. (i) Let $\mathscr{H}$ be the algebra of real valued rational functions of $(x, y) \in \Omega$, generated by

$$
\left.\operatorname{tr}\left[f(X) \cdot f\left(L_{\alpha}(x, y+\alpha x)\right)\right] \quad(\operatorname{tr} f(X)) \cdot \operatorname{tr} f\left(L_{\alpha}(x, y+\alpha x)\right)\right),
$$


where $f$ is a polynomial. Then every member of $\mathscr{H}$ is an invariant under the action of $\phi_{+}$.

(ii) Similarly let $\tilde{\mathscr{H}}$ be the algebra of real-valued rational functions generated by $\operatorname{tr}[f(X) \cdot f(L(x, y)), \quad(\operatorname{tr} f(X)) \cdot(\operatorname{tr} f(L(x, y)))$,

$f$ a polynomial. Then $\tilde{\mathscr{H}}$ is a list of rational invariants of $\psi_{+}$.

Proof. Let $\mathscr{M}$ be the algebra of matrix valued functions $M(x, y)$ of $(x, y)$, generated by $X=\operatorname{diag}\left(x_{1}, \ldots, x_{n}\right)$ and $L_{\alpha}^{T}(x, y+\alpha x)$. Note that any homomorphism of $\mathscr{M}$ is completely specified by its behavior on $X, L_{\alpha}^{T}(x, y+\alpha x)$, through extension. Now the map $\phi_{+}(x, y)=(p, q)$ induces a mapping

$$
\left(X, L_{\alpha}^{T}(x, y+\alpha x)\right) \rightarrow\left(L_{\alpha}(q, p+\alpha q), Q\right),
$$

explicitly given by (3.7a). By the above remark, this extends naturally to a homomorphism $\hat{\phi}_{+}: \mathscr{M} \rightarrow \mathscr{M}$, which by (3.7a) is expressed by

$$
\hat{\phi}_{+}(M(x, y))=\mathscr{U}^{-1}(x, y) M(x, y) \mathscr{U}(x, y) .
$$

Since $\phi_{+}(x, y)=(q, p)$, we can consider functions of $(x, y)$ as functions of $(q, p)$, which we shall do when we think of $\mathscr{M}$ as the range of the map $\hat{\phi}_{+}$, i.e., we shall regard $\hat{\phi}_{+}(M(x, y))$ as a function of $(q, p)$. We note that trace is an invariant of $\hat{\phi}_{+}$. Hence defining $\hat{f}(x, y)$ for any polynomial $f$ by the following line:

$$
\begin{aligned}
\hat{f}(x, y) & \equiv \operatorname{tr}\left[f(X) \cdot f\left(L_{\alpha}(x, y+\alpha x)\right)\right]=\operatorname{tr}\left(\hat{\phi}_{+}\left[f(X) \cdot f\left(L_{\alpha}^{T}(x, y+\alpha x)\right)\right]\right) \\
& =\operatorname{tr}\left[f\left(\hat{\phi}_{+}(X)\right) \cdot f\left(\hat{\phi}_{+}\left(L_{\alpha}^{T}(x, y+\alpha x)\right)\right)\right]=\operatorname{tr}\left[f\left(L_{\alpha}(q, p+\alpha q)\right) \cdot f(Q)\right] \\
& =\operatorname{tr}\left[f(Q) \cdot f\left(L_{\alpha}(q, p+\alpha q)\right)\right] \equiv \hat{f}(q, p),
\end{aligned}
$$

we find $\hat{f}(x, y)=\hat{f}(q, p)$ if $\varphi_{+}(x, y)=(q, p)$, i.e., $\hat{f}($,$) is an invariant. The other part of (\mathrm{i})$ is immediate, while (ii) is proven precisely as (i).

We remark that an alternate derivation of Theorem 7 can be given, whereby the invariance of the above quantities is seen to follow from the "invariance" of the Poisson bracket under canonical transformations, (see [14]).

\section{Periodicity of Solutions for $\alpha$ Purely Imaginary and a Perturbation Result}

By Theorem 3, the homogeneous symmetric polynomials of degree $v$ in the $x_{i}$ are polynomials of degree $v$ in $e^{ \pm \alpha t}$, for the system (A), $\alpha \neq 0$. On the other hand, the $x_{i}$ are strictly ordered, $x_{1}<x_{2}<\ldots<x_{n}$, due to the singularity in the potential, hence the $x_{i}$ are uniquely given as algebraic functions of $e^{ \pm \alpha t}$ (and the initial data). We thus conclude, verifying a conjecture of Calogero's [10]:

Corollary 3.2. If $(x, y)$ obey $\dot{x}_{i}=\partial H_{\alpha} / \partial y_{i}, \dot{y}_{i}=-\partial H_{\alpha} / \partial x_{i}, i=1, \ldots, n, \alpha$ pure imaginary, then the solutions are all periodic with (not necessarily primitive) period $2 \pi i \alpha^{-1}$.

Remark 1. Since the motion is periodic, one expects $2 n-1$ integrals of the motion to exist, which in this case would be rational in $(x, y)$, and indeed they do. Take for the first $n$ integrals $I_{j}[E]=\operatorname{tr} E^{j}$, and for the latter $n-1$ integrals, Real $\left[I_{j}\left(M^{+}\right) I_{1}^{j}\left(M^{-}\right)\right]$, $j=2, \ldots, n$. One sees they are algebraically independent by considering the $x_{i}$ far apart, in which case the matrices are nearly diagonal. 
Remark 2. A linearizing transformation, which is rational in one direction, algebraic in the other direction, is given $b^{3} \tau:(x, y) \rightarrow\left\{\operatorname{tr}(L+\alpha X)^{v}\right\}_{v=1}^{n}=\left(z_{1}, \ldots, \mathrm{z}_{n}\right)=z$, $(x, y) \in \Omega, \Omega$ defined at the beginning of Section 2 , and maps the flow into $\dot{z}=j \alpha \dot{z}_{j}$, (see [9]). The mapping $\tau$ may not be invertible, but for $x_{i+1}-x_{i}$ large, $L+\alpha X$ is close to $\operatorname{diag}\left(x_{1}+\alpha y_{1}, \ldots, x_{n}+\alpha y_{n}\right)$ and thus by the implicit function theorem, knowledge of $z$ enables us to recover $(x, y)$ from $z$ and $\bar{z}$. Hence $z_{1}, \ldots, z_{n}$ are algebraically independent, complex valued rational functions of $x, y$.

Theorem 8. For every sufficiently small $C^{2}$ perturbation of $H_{\alpha}$, there exists at least $n$ geometrically distinct periodic solutions on every energy surface $E>E_{\alpha}=\min _{(x, y)} H_{\alpha}(x, y)$.

While such results are easily derived in case the periodic solutions of the unperturbed system are isolated, they are quite delicate for manifolds of periodic orbits which we have encountered. We shall use Theorem 1.4 of [8], a beautiful theorem of Weinstein. Instead of stating Theorem 1.4 in its full generality, which requires much terminology [8], we shall just list an immediate consequence of it, which will suffice for our purpose, and then we shall verify that the necessary hypothesis are satisfied in our situation.

Corollary of Theorem 1.4. Given: The Hamiltonian system $H^{(\varepsilon)}=H+\varepsilon P(x, y, \varepsilon),(x, y)$ in a neighborhood of the manifold given by the relation $H^{(0)}=E$, and $\nabla H^{0} \neq 0$ on $H^{(0)}=E, P$ being $C^{2}$ in its arguments, while for the value $E, H^{(0)}=E$ is a manifold which is homotopic to the sphere, free of equilibria, of only periodic solutions. ${ }^{4}$ Then, for small $\varepsilon$, the system with $E=H^{(\varepsilon)}$ contains at least $n$ geometrically distinct periodic orbits. Proof of Theorem 8. We shall apply the corollary to the case $H=H_{\alpha}, \alpha$ purely imaginary, $E>E_{\alpha}=\min _{x, y} H_{\alpha}(x, y)$.

Thus we just have to check for $E>E_{\alpha}$, the manifold $H_{\alpha}=E$ is diffeomorphic (homotopic would suffice) to $S^{2 n-1}$, with no fixed points. Now

$$
H_{\alpha}=\frac{1}{2} \sum y_{1}^{2}+V_{\alpha}, \quad V_{\alpha}=-\frac{1}{2} \alpha^{2} \sum x_{i}^{2}+\sum_{i<j}\left(x_{i}-x_{j}\right)^{-2},
$$

and we see that $V_{\alpha}(x)$ is strictly convex, since

$$
\left(\eta, V_{x x} \eta\right)=\left(d^{2} / d t^{2}\right) V_{\alpha}(\xi+t \eta)=-\alpha^{2}\left(\sum \eta_{i}^{2}\right)+6 \sum\left(\eta_{i}-\eta_{j}\right)^{2} /\left(\xi_{i}-\xi_{j}\right)^{4}>0,
$$

for purely imaginary $\alpha$. Thus $V_{\alpha}(x)$ assumes a strict nondegenerate minimum at some unique $x_{\alpha}$, where $V_{\alpha}\left(x_{\alpha}\right)=E_{\alpha}$. We conclude $H_{\alpha}(x, y)$ is strictly convex in $(x, y) \in \Omega$, and now consider the surface $H_{\alpha}=E>E_{\alpha}$, which by the above contains no fixed points of the flow. The smooth surface $H_{\alpha}=E$ is thus the boundary of the convex body $H_{\alpha} \leqq E$, and hence is diffeomorphic to a sphere, the diffeomorphism being given by spherical projection through an interior point of the body to some large fixed sphere. Clearly $\nabla H_{\alpha} \neq 0$ on $H=E$, for $E>E_{\alpha}$.

Remark 3. For $n=2,3$, one can show that for energy surfaces near the equilibrium point, one has distinct orbits of primitive periods $2 \pi i \alpha^{-1} .\left\{1, z^{-1}, \ldots, n^{-1}\right\}$, and Gallavotti and Marchioro [7] conjectured this to be true for all $n$. Thus $H_{\alpha}$ is quite different from the system $H=\frac{1}{2} \sum y_{i}^{2}-\frac{1}{2} \alpha^{2} \sum x_{i}^{2}$, for which all solutions have the same primitive period.

3 This result is contained in a recent note by Sawada and Kotera, see [9], which, moreover, motivated Theorem 3

4 We assume here that all orbits have a common period to avoid technicalities 


\section{Associated Flows}

The integrals found for the system $H_{\alpha}$, Case (A), were of the form $\operatorname{tr} E^{p}$ (see Theorem 2). More generally $H^{f}=\operatorname{tr} f(E)$, for any polynomial $f$, gives rise to an integral. It is thus natural to consider $H^{f}$ as the Hamiltonian of a flow, which we call associated with $H_{\alpha}=\operatorname{tr} E$. For these more general flows $\operatorname{tr} E^{p}$ or also $\operatorname{tr} f(E)$ are, of course, again integrals since all these functions are in involution, and therefore $E$ is isospectral under all flows $H^{f}$. We will determine another isospectral matrix, which, however, depends explicitly on $t$. This will allow us, as in the case of the system $H_{\alpha}$, to describe the solutions of the system $H^{f}$ explicitly. The result is stated in Theorem 10 below.

We will determine these isospectral matrices, first for another flow, and later translate the results to the $H^{f}$ flow. For most of this section we will study this other system, which is closely related to the Sutherland system, (1.1), Case (B), $\alpha=0$, and carry out the above plan for it. The system to be considered now is given by the Hamiltonian:

$$
G(q, p)=\frac{1}{2} \sum_{i=1}^{n}\left(q_{i} p_{i}\right)^{2}+\frac{1}{4} \sum_{i<j}\left(q_{i}+q_{j}\right)^{2} /\left(q_{i}-q_{j}\right)^{2}, \quad(q, p) \in \Omega,
$$

$\Omega$ defined in Section 2 .

Notice that $G(q, p)$ is, after the trivial canonical change of coordinates (2.6), transformed into the system given by (2.8), i.e. $H_{\alpha}$ of (1.1) Case (B), $\alpha=0$. For the matrix description of the flow we introduce:

$$
\{R(q, p)\}_{j k}=\delta_{j k}\left(q_{j} p_{j}\right)+\frac{1}{2} i\left(1-\delta_{j k}\right)\left(q_{j}+q_{k}\right) /\left(q_{j}-q_{k}\right),
$$

$R^{ \pm}=R \pm C$ where $C_{j k}=\frac{1}{2} i\left(1-\delta_{j k}\right)$, and therefore

$$
\left\{\begin{array}{l}
R_{j k}^{+}(q, p)=\delta_{j k}\left(q_{j} p_{j}\right)+i\left(1-\delta_{j k}\right) q_{j} /\left(q_{j}-q_{k}\right) \\
R_{j k}^{-}(q, p)=\delta_{j k}\left(q_{j} p_{j}\right)+i\left(1-\delta_{j k}\right) q_{k} /\left(q_{j}-q_{k}\right)
\end{array}\right\} .
$$

We observe that $R(q, p)=Z(\xi,-\eta), R^{ \pm}=Z^{ \pm}$, with $Z, Z^{ \pm}$defined in (2.7), and $(q, p) \rightarrow(\xi, \eta)$ defined in $(2.6)$ for $q_{i}>0, i=1,2 \ldots, n$. Thus by the discussion immediately following (2.10), $R^{ \pm}$, for the case $q_{i}>0, i=1,2, \ldots, n$, has simple spectrum. We note that by definition $\left(R^{+}\right)^{*}=R^{-}, R^{+} Q=Q R^{-}$, where $Q=\operatorname{diag}\left(q_{1}, q_{2}, \ldots, q_{n}\right)$. Thus it is clear that $R^{+}, R^{-}$have the same spectrum if $Q$ is nonsingular, or equivalently that $\operatorname{tr}\left(R^{+}\right)^{v}=\operatorname{tr}\left(R^{-}\right)^{v}$, for all $v$. Since the latter equation is rational in $(q, p)$, it holds for all $(q, p) \in \Omega$. Similar $Q R^{-}=R^{+} Q$ implies $Q f^{\prime}\left(R^{-}\right)=f^{\prime}\left(R^{+}\right) Q$ for $f^{\prime}$ a polynomial, and so

$$
q_{i}\left[f^{\prime}\left(R^{-}\right)\right]_{i j}=\left[f^{\prime}\left(R^{+}\right)\right]_{i j} q_{j} .
$$

If $f^{\prime}$ is real, then $\left[Q f^{\prime}\left(R^{-}\right)\right]^{*}=\left[f^{\prime}\left(R^{-}\right)\right]^{*} Q^{*}=f^{\prime}\left(R^{+}\right) Q=Q f^{\prime}\left(R^{-}\right)$, i.e., $Q f^{\prime}\left(R^{-}\right)$as well as $f^{\prime}\left(R^{+}\right) Q$ are Hermitian, and so in particular $q_{i}\left[f^{\prime}\left(R^{-}\right)\right]_{i i}$ as well as $q_{i}\left[f^{\prime}\left(R^{+}\right)\right]_{i i}$, and $\left[f^{\prime}\left(R^{-}\right)\right]_{i i}=\left[f^{\prime}\left(R^{+}\right)\right]_{i i}$ are real. Thus we conclude

$$
\left\{\begin{array}{l}
i \sum_{k \neq j}\left[f^{\prime}\left(R^{+}\right)\right]_{j k} R_{k j}^{+}=i\left\{\left[f^{\prime}\left(R^{+}\right) R^{+}\right]_{j j}-\left[f^{\prime}\left(R^{+}\right)\right]_{j j} R_{j j}^{+}=i\left\{\left[g\left(R^{+}\right)\right]_{j j}\right.\right. \\
\left.-q_{j} p_{j}\left[f^{\prime}\left(R^{+}\right)\right]_{j j}\right\}
\end{array}\right\}
$$

with $g(s)=s f^{\prime}(s)$, is purely imaginary. 
An easy calculation shows that for $G(q, p)$ defined in (5.1),

$$
G(q, p)=\operatorname{tr}\left(\frac{1}{2}\left(R^{-}\right)^{2}+c I\right)=\operatorname{tr}\left(\frac{1}{2}\left(R^{+}\right)^{2}+c I\right), \quad c=-n^{2}(n-1) / 8 .
$$

Theorem 9. Under the flow $\dot{q}_{i}=\partial F / \partial p_{i}, p_{i}=-\partial F / \partial q_{i}$, with the Hamiltonian

$$
F=F^{f}=\operatorname{tr} f\left(R^{+}\right)=\operatorname{tr} f\left(R^{-}\right),
$$

which is associated with $G(q, p)$ of $(5.1)$, the symmetric polynomials in $q_{i}=q_{i}(t)$ are rational in exponentials $e^{\lambda j t}, j=1, \ldots, n$, provided $q_{j}>0$. This is a consequence of the fact that the four matrices,

$$
R^{ \pm}, M^{+}=e^{-t f^{\prime}\left(R^{+}\right)} Q, \quad M^{-}=Q e^{-t f^{\prime}\left(R^{-}\right)},
$$

undergo isospectral deformations as $(q, p)=(q(t), p(t))$ evolve under the $F$ flow.

Proof. These matrices satisfy an equation of the form $\dot{L}=[A, L]$, where $A=A^{+}=A^{-}, A=-A^{*}, A^{ \pm}$defined by the two equivalent relations

$$
\left\{\begin{array}{l}
A_{j k}^{-}(q, p)=\delta_{j k}\left(-\sum_{s \neq k} i\left[f^{\prime}\left(R^{-}\right)\right]_{s k} R_{k s}^{-}\right)+i\left(1-\delta_{j k}\right)\left[f^{\prime}\left(R^{-}\right)\right]_{j k} R_{k j}^{-} \\
A_{j k}^{+}(q, p)=\delta_{j k}\left(-\sum_{s \neq k} i\left[f^{\prime}\left(R^{+}\right)\right]_{s k} R_{k s}^{+}\right)+i\left(1-\delta_{j k}\right)\left[f^{\prime}\left(R^{+}\right)\right]_{j k} R_{k j}^{+}
\end{array}\right\},
$$

The identity of $A^{+}, A^{-}$is an immediate consequence of (5.4), (5.3), while $A=-A^{*}$ follows from the fact that $f\left(R^{+}\right) Q$, hence $Q f\left(R^{-}\right)$is Hermitian, and from (5.5). To prove the matrices of (5.6) are isospectral, it is sufficient to prove
(a) $\dot{R}^{+}=\left[A^{-}, R^{+}\right]$
(b) $\dot{M}^{-}=\left[A^{-}, M^{-}\right]$,

because taking the adjoint of (5.8), the fact $A=A^{ \pm}=-A^{*}$ implies a statement analogous to (5.8) for $R^{-}, M^{+}$. We first prove (a), i.e., $D_{F} R^{+}=\left[A^{-}, R^{+}\right]$. Using (5.3), and defining $\left[f^{\prime}\left(R^{-}\right)\right]_{i j}=t_{i j}$, we make a few preliminary calculations:

$$
\left\{\begin{array}{l}
D_{F} q_{s}=\partial_{p_{s}}\left[\operatorname{tr} f\left(R^{-}\right)\right]=\operatorname{tr}\left[f^{\prime}\left(R^{-}\right) \cdot \partial_{p_{s}} R^{-}\right]=q_{s} t_{s s} \\
D_{F} p_{s}=-\partial_{q_{s}}\left[\operatorname{tr} f\left(R^{-}\right)\right]=-p_{s} t_{s s}+i \sum_{j \neq s} \frac{q_{j}\left(t_{j s}-t_{s j}\right)}{\left(q_{s}-q_{j}\right)^{2}}
\end{array}\right\}
$$

where we have used $\left[\partial_{q_{s}} R^{-}\right]_{j k}=\delta_{j k}\left(p_{j} \delta_{j s}\right)+\frac{i\left(1-\delta_{j k}\right)}{\left(q_{j}-q_{k}\right)^{2}}\left(q_{j} \delta_{k s}-q_{k} \delta_{j s}\right)$. Hence $D_{F} R_{k k}^{+}$

$$
\begin{aligned}
& =\left(q_{k} p_{k}\right)^{\cdot}=\dot{q}_{k} p_{k}+q_{k} \dot{p}_{k}=i \sum_{j \neq k} \frac{q_{j} q_{k}}{\left(q_{j}-q_{k}\right)^{2}}\left(t_{j k}-t_{k j}\right) . \text { But } \\
& \begin{aligned}
{\left[A^{-}, R^{+}\right]_{k k} } & =\sum_{j \neq k}\left(A_{k j}^{-} R_{j k}^{+}-R_{k j}^{+} A_{j k}^{-}\right) \\
= & i\left[\sum_{j \neq k}\left\{\left(\frac{q_{k} t_{k j}}{q_{k}-q_{j}}\right) \cdot\left(\frac{q_{j}}{q_{j}-q_{k}}\right)-\left(\frac{q_{k}}{q_{k}-q_{j}}\right)\left(\frac{q_{j} t_{j k}}{q_{j}-q_{k}}\right)\right\}\right] \\
= & i \sum_{j \neq k}\left(\frac{q_{j} q_{k}}{\left(q_{j}-q_{k}\right)^{2}}\right)\left(t_{j k}-t_{k j}\right),
\end{aligned}
\end{aligned}
$$

and so we have shown $D_{F} R_{k k}^{+}=\left[A^{-}, R^{+}\right]_{k k}$, and we now must show $D_{F} R_{j k}^{+}=\left[A^{-}, R^{+}\right]_{j k}, j \neq k$. 
We compute

$$
\begin{aligned}
D_{F} R_{j k}^{+} & =D_{F} \frac{i q_{j}}{\left(q_{j}-q_{k}\right)} \\
& =i\left(\frac{q_{j} t_{j j}}{\left(q_{j}-q_{k}\right)}-\frac{q_{j}\left(q_{j} t_{j j}-q_{k} t_{k k}\right)}{\left(q_{j}-q_{k}\right)^{2}}\right)=\frac{i q_{j} q_{k}\left(t_{k k}-t_{j j}\right)}{\left(q_{j}-q_{k}\right)^{2}},
\end{aligned}
$$

hence

$$
\begin{aligned}
& q_{j} \Delta_{j k} /\left(q_{j}-q_{k}\right) \\
& \equiv\left[A^{-}, R^{+}\right]_{j k}-D_{F} R_{j k}^{+} \text {(defining } \Delta_{j k} \text { by this equation) } \\
& =\sum_{s}\left(A_{j s}^{-} R_{s k}^{+}-R_{j s}^{+} A_{s k}^{-}\right)-D_{F} R_{j k}^{+} \\
& =\left[\frac{q_{j} t_{j k}}{\left(q_{j}-q_{k}\right)}\right]\left(R_{k k}^{+}-R_{j j}^{+}\right)+\left(A_{j j}^{-}-A_{k k}^{-}\right)\left[\frac{i q_{j}}{\left(q_{j}-q_{k}\right)}\right] \\
& \quad+\left\{i \sum_{s \neq j, k}\left[\left[\frac{q_{j} t_{j s}}{\left(q_{j}-q_{s}\right.}\right] \cdot\left[\frac{q_{s}}{\left(q_{s}-q_{k}\right)}\right]-\left[\frac{q_{j}}{\left(q_{j}-q_{s}\right)}\right] \cdot\left[\frac{t_{s k} q_{s}}{\left(q_{s}-q_{k}\right)}\right]\right]\right\} \\
& \quad-i q_{j} q_{k} \frac{\left(t_{k k}-t_{j j}\right)}{\left(q_{j}-q_{k}\right)^{2}} .
\end{aligned}
$$

We now wish to show $\Delta_{j k}=0$, and for that we shall use the addition law,

$$
\frac{\left(q_{j}-q_{k}\right)}{\left(q_{j}-q_{s}\right)\left(q_{s}-q_{k}\right)}=\frac{1}{\left(q_{j}-q_{s}\right)}+\frac{1}{\left(q_{s}-q_{k}\right)},
$$

inside the bracketed term. So we compute

$$
\begin{aligned}
\Delta_{j k}= & t_{j k}\left(R_{k k}^{+}-R_{j j}^{+}\right)+i\left(A_{j j}^{-}-A_{k k}^{-}\right)+i q_{k} \frac{\left(t_{j j}-t_{k k}\right)}{\left(q_{j}-q_{k}\right)} \\
& +i \sum_{s \neq j, k}\left\{\left(t_{j s}-t_{s k}\right)\left[\frac{q_{s}}{\left(q_{j}-q_{s}\right)}+\frac{q_{s}}{\left(q_{s}-q_{k}\right)}\right]\right\} .
\end{aligned}
$$

We compute the bracketed term separately:

$$
\begin{aligned}
& \left(t_{j s}-t_{s k}\right)\left[\frac{q_{s}}{\left(q_{j}-q_{s}\right)}+\frac{q_{s}}{\left(q_{s}-q_{k}\right)}\right] \\
& =\frac{t_{j s} q_{s}}{\left(q_{j}-q_{s}\right)}-\frac{t_{s k} q_{s}}{\left(q_{s}-q_{k}\right)}+\frac{t_{j s} q_{s}}{\left(q_{s}-q_{k}\right)}-\frac{t_{s k} q_{s}}{\left(q_{j}-q_{s}\right)} \\
& =\left[-t_{j s}+\frac{q_{j} t_{j s}}{\left(q_{j}-q_{s}\right)}\right]-\frac{q_{s} t_{s k}}{\left(q_{s}-q_{k}\right)}+\left[t_{j s}+\frac{q_{k} t_{j s}}{\left(q_{s}-q_{k}\right)}\right]-\frac{q_{s} t_{s k}}{\left(q_{j}-q_{s}\right)} \\
& =A_{j s}^{-}-A_{s k}^{-}-i\left(t_{j s} R_{s k}^{-}-R_{j s}^{-} t_{s k}\right) .
\end{aligned}
$$


Hence

$$
\begin{aligned}
\Delta_{j k}= & i\left[\sum_{s} A_{j s}^{-}-\sum_{s} A_{s k}^{-}\right]+\sum_{s \neq j, k}\left(t_{j s} R_{s k}^{-}-R_{j s}^{-} t_{s k}\right) \\
& +t_{j k}\left(R_{k k}^{-}-R_{j j}^{-}\right)+R_{j k}^{-}\left(t_{j j}-t_{k k}\right) \\
= & i\left[\sum_{s} A_{j s}^{-}-\sum_{s} A_{s k}^{-}\right]+\left[f^{\prime}\left(R^{-}\right), R^{-}\right]_{j k} \\
= & i\left[\sum_{s} A_{j s}^{-}-\sum_{s} A_{s k}^{-}\right]=i \sum_{s} A_{j s}^{-}, \quad \text { by }
\end{aligned}
$$

So we just have to show $i \sum_{s} A_{j s}^{-}=0$, i.e., that $A^{-} \tilde{C}=0$, where $\tilde{C}_{j k}=i$ for all $j, k$. But since $\sum_{s} A_{s k}^{-}=0$, for all $k$, we have $\tilde{C} A^{-}=0$, and so $0=\left(\tilde{C} A^{-}\right)^{*}=A^{-} \tilde{C}$, as was to be shown, concluding the proof of $(5.8 \mathrm{a})$. It remains to prove (5.8b) i.e., $\dot{M}^{-}=\left[A^{-}, M^{-}\right]$.

We first compute

$$
\begin{aligned}
\left(M^{-}\right)^{\cdot} & =\left(Q e^{-t f^{\prime}\left(R^{-}\right)}\right)^{\cdot} \\
& =\left(D_{F} Q\right) e^{-t f^{\prime}\left(R^{-}\right)}+Q\left(e^{-t f^{\prime}\left(R^{-}\right)}\right)^{\cdot} \\
& =\left(D_{F} Q\right) e^{-t f^{\prime}\left(R^{-}\right)}+Q\left\{-f^{\prime}\left(R^{-}\right) e^{-t f^{\prime}\left(R^{-}\right)}+\left[A^{-}, e^{-t f^{\prime}(R)}\right]\right\},
\end{aligned}
$$

where we have made use of $D_{F} R^{-}=\left[A^{-}, R^{-}\right]$, which implies $D_{F} g\left(R^{-}\right)$ $=\left[A^{-}, g\left(R^{-}\right)\right]$. On the other hand,

$$
\left[A^{-}, M^{-}\right]=\left[A^{-}, Q e^{-t f^{\prime}\left(R^{-}\right)}\right]=Q\left[A^{-}, e^{-t f^{\prime}\left(R^{-}\right)}\right]+\left[A^{-}, Q\right] e^{-t f^{\prime}\left(R^{\prime}\right)},
$$

and thus in order to have $\dot{M}^{-}=\left[A^{-}, M^{-}\right]$, we must have

$$
D_{F} Q=Q f^{\prime}\left(R^{-}\right)+\left[A^{-}, Q\right] \text {. }
$$

In other words, we must have

$$
\begin{aligned}
& D_{F} q_{j}=q_{j} t_{j j} \\
& {\left[Q, A^{-}\right]_{j k}\left(1-\delta_{j k}\right)=\left(q_{j}-q_{k}\right) A_{j k}^{-}\left(1-\delta_{j k}\right)=q_{j} t_{j k}\left(1-\delta_{j k}\right),}
\end{aligned}
$$

but (5.11) is an immediate consequence of (5.9), (5.7), and (5.3). We have thus proven (5.8), and the statement of the theorem concerning the rational character of the solutions will be easily shown to follow from

$$
Q(t)=W(t) Q_{0} e^{t f^{\prime}\left(R_{0}^{-}\right)} W^{-1}(t),
$$

where $W$ is a matrix satisfying $W_{0}=I, \dot{W}=A^{-} W$, which we quickly verify. Indeed, as a consequence of (5.8),

$$
M^{-}=Q e^{-t f^{\prime}\left(R^{-}\right)}=W Q_{0} W^{-1}, \quad R^{-}=W R_{0}^{-} W^{-1},
$$

where the subscript shall now, and in the future indicate evaluation at $t=0$, and no subscript indicates evaluation at $t$. Hence

$$
Q=W Q_{0} W^{-1} e^{t f^{\prime}\left(R^{-}\right)}=W Q_{0} W^{-1}\left(W e^{t f^{\prime}\left(R_{\overline{0}}^{-}\right)} W^{-1}\right)=W Q_{0} e^{t f^{\prime}\left(R_{\overline{0}}^{-}\right)} W^{-1} .
$$

We note that Olshanetsky and Perelomov (see Footnote 2) have proven (5.12) for $f(s)=\frac{1}{2} s^{2}$, in slightly different coordinates. 
The rational character of the solutions now follows, for by previous remarks, $R^{ \pm}$has simple spectrum $v_{1}<v_{2}<\ldots<v_{n}$ for $0<q_{1}<q_{2}<\ldots<q_{n}$, and so by (5.12),

$$
\operatorname{tr} Q^{v}=\sum_{i=1}^{n} q_{i}^{v}=P_{v}\left(e^{f^{\prime}\left(v_{s}\right) t}\right),
$$

where $P_{v}$ is a polynomial of degree $v$ in the $n$ exponentials $e^{f^{\prime}\left(v_{s}\right) t}$.

Remark 1. In the language of Remark 1 of Section 2, the matrix equations of motion under the $F$ flow may be expressed as:
(a) $\delta R^{ \pm}=0$,
(b) $\delta Q=Q f^{\prime}\left(R^{-}\right)=f^{\prime}\left(R^{+}\right) Q$
(c) $\delta M^{ \pm}=0$,

where the derivation $\delta$ is defined by

$$
\delta(\cdot)=D_{F}(\cdot)-[A,(\cdot)]+\partial_{t}(\cdot) .
$$

Remark 2. If we introduce $x_{j}, y_{j}$, by $q_{j}=e^{i x_{j}}, p_{j}=-i y, j=1, \ldots, n$, a canonical transformation, then $i R_{j k}$ becomes

$$
\delta_{j k} y_{j}+\frac{1}{2} i\left(1-\delta_{j k}\right) \cot \frac{1}{2}\left(x_{j}-x_{k}\right) \text {. }
$$

If we take $f(s)=\frac{1}{2} s^{2}$ in Theorem 9 , then all solutions are bounded as a consequence of

$$
-\frac{1}{2} \operatorname{tr} R^{2}=\frac{1}{2} \sum_{i=1}^{n} y_{i}^{2}+\frac{1}{4} \sum_{i<j} \cot ^{2} \frac{1}{2}\left(x_{i}-x_{j}\right)
$$

being a constant of the motion. To be more specific, we also observe that $R_{0}^{-}$is diagonalizable, since $Q=W Q_{0} e^{t R_{0}^{-}} W^{-1}$, with $W$ unitary, $Q=\operatorname{diag}\left(e^{i x_{1}}, \ldots, e^{i x_{n}}\right)$, and thus the matrix $e^{t R_{0}^{-}}$is bounded for all real $t$. Write $e^{t R_{0}^{-}}$in diagonalized form and observe that since $e^{t R_{0}^{-}}$is bounded, $R_{0}^{-}$has purely imaginary, not necessarily distinct eigenvalues $i v_{1}, \ldots, i v_{n}$. From which we conclude, using (5.12), that

$$
Q \sim S e^{i D(v)}=\sum_{j=1}^{n} S^{j} e^{i v_{j} t}
$$

where $D(v)=\operatorname{diag}\left(v_{1}, \ldots, v_{n}\right)$, and the $S^{j}$ 's are the column vectors of $S$. Since necessarily, $|\operatorname{det} S|=1 \neq 0$, we must have $S^{j} \neq 0$ for all $j$. Thus all solutions of the flow

$$
\begin{aligned}
& \dot{x}_{i}=H_{y_{i}}, \quad \dot{y}_{i}=-H_{x_{i}}, \quad i=1, \ldots, n, \\
& H=\frac{1}{2} \sum_{j=1}^{n} y_{j}^{2}+\frac{1}{4} \sum_{i<j} \cot ^{2} \frac{1}{2}\left(x_{i}-x_{j}\right)
\end{aligned}
$$

are quasi-periodic; and moreover, in special cases they will be periodic, namely if the $v_{j}$ are integer multiples of a number $\mu \neq 0$.

The quasi-periodic character of the solutions would be a consequence of a well known theorem of Arnold [6], if only we knew that the gradiants of the $n$ integrals of the compact system $H=\frac{1}{2} \operatorname{tr} R^{2}$ were everywhere linearly independent. Since we don't know this, it is conceivable that such phenomena as exceptional points, hyperbolic tori, etc., occur, which is ruled out by the quasi-periodic behavior of the solutions. Of course, the rational character of the solutions is a stronger assertion. 
Corollary 9.1. Under the same hypothesis as Theorem 9 , with $f(s)$ replaced by $f(-\alpha s)$; i.e., $H^{f}=\operatorname{tr} f\left(-\alpha\left(R^{ \pm}\right)^{T}\right)$, we have for the time evolution of $(q, p)=(q(t), p(t))$, with the Hamiltonian $H^{f}$,

$$
\begin{aligned}
& L_{\alpha}(q, q+\alpha p) \\
& =W\left[2^{-1 / 2}\left\{Q_{0} e^{-\alpha t f^{\prime}\left(-\alpha R_{0}\right)}+\alpha_{-}^{-1} e^{\alpha t f^{\prime}\left(-\alpha R_{0}^{\overline{0}}\right)} L\left(q_{0}, p_{0}\right)\right\}\right] W^{-1} .
\end{aligned}
$$

Proof. Since $Q L(q, p)=R^{+}, Q=W Q_{0} e^{-\alpha t f^{\prime}\left(-\alpha R_{0}^{-}\right)} W^{-1}, R^{+}=W R_{0}^{+} W^{-1}$ imply $L(q, p)=W e^{\alpha t f^{\prime}\left(-\alpha R_{0}^{-}\right)} L\left(q_{0} p_{0}\right) W^{-1}$, we get from $L_{\alpha}(q, p+\alpha q)=2^{-1 / 2}\left(Q+\alpha^{-1} L(q, p)\right)$, by substituting the above expressions, the stated result.

Now we return to our original family of systems $H^{f}=\operatorname{tr}(f(E(x, y))$, and the plan of the beginning of this section. The tool is simply the canonical transformation used to prove Theorem 2, which takes $F^{f}$ into $H^{f}$.

Theorem 10. If $\dot{x}_{i}=\partial_{y_{i}} H^{f}(x, y), \dot{y}_{i}=-\partial_{x_{i}} H^{f}(x, y), i=1, \ldots, n$, then the three matrices

$$
E, e^{-\alpha t f^{\prime}(E)}(Y+\alpha X), \quad(Y-\alpha X) e^{\alpha t f^{\prime}(E)},
$$

undergo an isospectral deformation with the same unitary generator for all the deformations, and we have the time dependence of the solutions given by

$$
X(t) \sim \frac{1}{2} \alpha^{-1}\left\{e^{\alpha f^{\prime}\left(E_{0}\right) t}\left(Y_{0}+\alpha X_{0}\right)+\left(\alpha X_{0}-Y_{0}\right) e^{-\alpha f^{\prime}\left(E_{0}\right) t}\right\},
$$

where $\sim$ indicates unitary equivalence.

Proof. By (3.5), (3.10), and $M^{ \pm}=(Y \pm \alpha X) e^{\mp \alpha t}$, we have for $\alpha>0$,

$$
\hat{\phi}_{+}:\{Y+\alpha X,-Y+\alpha X\} \rightarrow\left\{2^{1 / 2} \alpha Q, 2^{1 / 2} L^{T}(q, p)\right\},
$$

$\hat{\phi}_{+}$discussed briefly in the proof of Theorem 7 . We recall that $\hat{\phi}_{+}$acted on the ring $\mathscr{M}$ of matrix valued functions of the variables $(x, y)=\phi_{+}^{-1}(q, p)$, generated by $Y+\alpha X, Y-\alpha X$, by extending formula (5.16), so that for $M(x, y) \in \mathscr{M}$, we have $\hat{\phi}(M(x, y))=\mathscr{U}^{-1}(x, y) M(x, y) \mathscr{U}(x, y)$. As a consequence of the definition of $\hat{\phi}_{+}$, we found in (3.6a)

$$
\hat{\phi}_{+}(X)=L_{\alpha}(q, p+\alpha q) \text {, }
$$

and

$$
\hat{\phi}_{+}(E)=-\alpha\left(R^{-}\right)^{T}, \text { where } E=\frac{1}{2}(Y+\alpha X)(Y-\alpha X) .
$$

We also note that the mapping $\phi_{+}$is canonical with multiplier -1 , so we replace $t$ by $-t$ in the change of coordinates $(q, p) \rightarrow(x, y)$, to describe evolution in time. Now we apply the inverse $\left(\hat{\phi}_{+}\right)^{-1}$ of the above transformation to derive from $(5.16),(5.17)$ that

$$
\begin{aligned}
\left(\hat{\phi}_{+}\right)^{-1}\left(Q e^{\alpha t f^{\prime}\left(-\alpha R^{-}\right)}\right)^{T} & =\left(\hat{\phi}_{+}\right)^{-1}\left(e^{\alpha t f^{\prime}\left(-\alpha\left(R^{-}\right)^{T}\right)} Q\right) \\
& =e^{\alpha(-t) f^{\prime}(E)} \cdot\left(2 \alpha^{2}\right)^{-1 / 2}(Y+\alpha X) \\
& =\left(2 \alpha^{2}\right)^{-1 / 2} e^{-\alpha t f^{\prime}(E)}(Y+\alpha X) .
\end{aligned}
$$

But by Theorem 9, $Q e^{\alpha t f^{\prime}\left(-\alpha R^{-}\right)}$is similar to $Q_{0}$, hence also to $(2 \alpha)^{-1 / 2}\left(Y_{0}+\alpha X_{0}\right)$. This shows that $e^{-\alpha t f^{\prime}(E)}(Y+\alpha X)$ is similar to $\left(Y_{0}+\alpha X_{0}\right)$, and thus is an isospectral matrix for the flow $H^{f}$. Formula (5.17) states $E$ is similar to $-\alpha\left(R^{-}\right)^{T}$, which by 
Theorem 9 is similar to $-\alpha\left(R_{0}^{-}\right)^{T}$, hence $E$ is isospectral. Formula (5.15) then follows similarly from (3.16a) and Corollary 9.1 .

At first Theorem 10 is valid only for $\alpha>0$, but as all the relations involved are algebraic, we may take $\alpha$ arbitrary. The formula for $X$ in Theorem 10 yields the explicit solutions of the motion, and as in Theorem 9, we conclude

$$
\operatorname{tr}(L \pm \alpha X)^{v}=P_{v}^{ \pm}\left(e^{ \pm \alpha t f^{\prime}\left(v_{s}\right)}\right),
$$

for $H=H^{f}$ (see Theorem 9), the $v_{i}$ 's distinct, for $\alpha>0$.

Corollary 10.1. If $H=H^{f}=\operatorname{tr} f(E)$, the differential equations of motion, written in matrix form are

(a) $\delta E=0, \quad$ (b) $\delta X=\frac{1}{2} \alpha\left[f^{\prime}(E), X\right]+\frac{1}{2}\left[f^{\prime}(E), Y\right]_{+}$,

with $[A, D]_{+}=A D+D A, \delta(\cdot)=D_{H}(\cdot)+\partial_{t}(\cdot)-[B,(\cdot)]$, where

$$
\left\{\begin{array}{l}
\text { a) } B_{j k}=-\frac{1}{2}\left\{\alpha\left[f^{\prime}(E)\right]_{j k}+\left(\left[f^{\prime}(E), Y\right]_{+}\right)_{j k} /\left(x_{j}-x_{k}\right)\right\}, j \neq k, \\
\text { b) } \sum_{s=1}^{n} B_{s j}=0
\end{array}\right\} \text {. }
$$

Proof. From Theorem 10 we have

$$
E, M^{+}=e^{-\alpha t f^{\prime}(E)}(Y+\alpha X), \quad M^{-}=(Y-\alpha X) e^{\alpha t f^{\prime}(E)}
$$

are isospectral under the $H^{f}$ flow, with one and the same unitary generator. In other words,

$$
\delta M^{ \pm}=0, \quad \delta E=0 .
$$

for some $B(t)$, which depends on initial data, which for the moment we use in the above definition of $\delta$. On the other hand, $\delta M^{+}=0$ implies

$$
\delta M^{+}=e^{-\alpha t f^{\prime}(E)} \delta(Y+\alpha X)+e^{-\alpha t f^{\prime}(E)}\left(-\alpha f^{\prime}(E)\right)(Y+\alpha X)=0,
$$

from which we conclude

$$
\delta(Y+\alpha X)=\alpha f^{\prime}(E)(Y+\alpha X)
$$

and similarly from $\delta M^{-}=0$, it follows

$$
\delta(Y-\alpha X)=-\alpha(Y-\alpha X) f^{\prime}(E) .
$$

From (5.20), (5.21), we compute

$$
\begin{aligned}
\delta E & =\delta\left[\frac{1}{2}(Y+\alpha X)(Y-\alpha X)\right] \\
& =\frac{1}{2}(\delta(Y+\alpha X))(Y-\alpha X)+\frac{1}{2}(Y+\alpha X)(\delta(Y-\alpha X)) \\
& =\frac{1}{2} \alpha f^{\prime}(E)(Y+\alpha X)(Y-\alpha X)-\frac{1}{2} \alpha(Y+\alpha X)(Y-\alpha X) f^{\prime}(E) \\
& =\alpha\left[f^{\prime}(E), E\right]=0 .
\end{aligned}
$$

Formula (5.19a) follows upon subtraction of (5.20) from (5.21), upon writing out the full expression for $\delta$.

We need only show that we can impose the normalization of (5.19b), which as we saw in Remark 2 of Section 2, which we shall be constantly referring to, is equivalent 
to requiring that the unitary generator of the matrix flow is contained in the Lie subgroup of the unitary group specified by $C U=C$, i.e., with Lie algebra specified by $C B=0$. From the proof of Theorem 10 , it follows that the unitary generator of the $H^{f}$ flow can be written as the product of the unitary generator of the $F^{f}$ flow of Theorem 9, and the U's occurring in the map $\hat{\phi}_{\alpha}$ of (5.16), as displayed in (3.10). By Remark 2, the latter $U$ 's [see the discussion preceding (3.5)] are contained in the above Lie group. The $U$ of the $F^{f}$ flow has as its infinitesimal generator the $A$ defined in (5.7), which clearly satisfies $C A=0$, and thus by Remark 2, this $U$ also is contained in the above Lie group. Thus the unitary generator of the $H^{f}$ flow can be written as the product of elements in the above specified Lie group, and so by Remark 2, we can impose the normalization condition (5.19b). We also observe that the fact $B(t)=B(x(t), y(t))$, i.e. that $B$ is really a function on $\Omega$, was not assumed a priori.

Remark 3. For $H=H^{f}=\operatorname{tr} f(Y), \alpha=0,(5.18)$ reduces to

$$
\delta Y=0, \quad \delta X=f^{\prime}(Y),
$$

where $\delta(\cdot)=D_{H}(\cdot)+\partial_{t}(\cdot)-[B,(\cdot)]$.

We also note that (5.22), (5.19) imply

$$
\begin{gathered}
\delta Y=0, \quad \delta\left(X-t f^{\prime}(Y)\right)=0, \\
\left\{\begin{array}{l}
B_{j k}=\left[f^{\prime}(Y)\right]_{j k} /\left(x_{j}-x_{k}\right), \quad j \neq k, \\
\sum_{j} B_{j s}=0
\end{array}\right\}
\end{gathered}
$$

and so by (5.23), the solutions of the $H^{f}$ flow obey $X(t) \sim\left(X_{0}+t f^{\prime}\left(Y_{0}\right)\right)$, hence

$$
\sum_{i=1} x_{i}^{v}(t)=\operatorname{tr}\left(X_{0}+t f^{\prime}\left(Y_{0}\right)\right)^{v}, \quad v=1, \ldots, \mathrm{n},
$$

the latter equation being a generalization of a result of Sawada and Kotera [9].

\section{Another Integrable System}

In this section we discuss another integrable system, namely (1.1) Case (B), $\alpha \neq 0$, formally analogous to Case (A), but physically behaving like a system of interacting particles under the influence of an additional force acting from the right. We shall make this more precise in the discussion of the scattering theory of the system. Moreover, we shall show the solutions of the system to be rational in exponentials by getting a fairly explicit formula for the time evolution of the system.

Theorem 11. The Hamiltonian system

$$
H_{\alpha}(x, y)=\frac{1}{2} \sum_{i=1}^{n} y_{i}^{2}+\frac{1}{4} \sum_{1 \leqq i<j \leqq n} \sinh ^{-2}\left(\frac{1}{2}\left(x_{i}-x_{j}\right)\right)+\alpha \sum_{i=1}^{n} e^{x_{i}}
$$

is integrable. If $\dot{x}_{i}=\partial H_{\alpha} / \partial y_{i}, \dot{y}_{i}=-\partial H_{\alpha} / \partial x_{i}, i=1, \ldots, n$, then the matrix

$$
W=\frac{1}{2} Z^{+}(x, y) Z^{-}(x, y)+\alpha e^{X}, \quad e^{X}=\operatorname{diag}\left(e^{x_{1}}, \ldots, e^{x_{n}}\right)
$$


undergoes an isospectral deformation

$$
D_{F_{\alpha}} W=[K, W] \text {, where } Z^{ \pm}=Z \pm \frac{1}{2} C \text {, }
$$

with $Z(x, y), C, K(x)$ as defined in (2.7), (2.9). Then the $n$ algebraically independent functions, $\tau_{j}=\operatorname{tr} W^{j}, j=1, \ldots, n$, rational in $\left(y_{i}, e^{x_{i}}\right)$, are in involution.

Proof. We shall actually prove this result for the system

$$
F_{\alpha}(q, p)=\frac{1}{2} \sum_{i=1}^{n}\left(q_{i} p_{i}\right)^{2}+\frac{1}{4} \sum_{1 \leqq i<j \leqq n}\left(q_{i}+q_{j}\right) /\left(q_{i}-q_{j}\right)+\alpha \sum q_{i}, \quad(q, p) \in \Omega,
$$

where $q$ is not necessarily positive, and then perform the usual canonical transformation,

$$
q_{i}=e^{x_{i}}, q_{i} p_{i}=y_{i}, \text { in the component of } \Omega \text { with } q_{i}>0 \text { for all } i .
$$

We then have

$$
Z^{ \pm}(x, y)=R^{ \pm}(q, p), \quad e^{X}=Q, \quad K(x)=A(q),
$$

with $R, Q, A$ of Theorem $9, f(s)=\frac{1}{2} s^{2}$. We compute the matrix differential equations of motion, using the derivation $\delta(\cdot)=D_{F_{\alpha}}(\cdot)-[A,(\cdot)]$, namely :

(a) $\delta Q=\frac{1}{2}\left(Q R^{-}+R^{+} Q\right)$,

(b) $\delta R^{ \pm}=-\alpha Q$.

Equation (6.4a) is the same equation you would get for $\alpha=0$, namely Equation (5.13b), for $f(s)=\frac{1}{2} s^{2}$, since $F_{0}=\frac{1}{2} \operatorname{tr}\left(R^{-}\right)^{2}, Q R^{-}=R^{+} Q$, and $D_{F_{\alpha}} q_{i}=\partial_{p_{i}} F_{\alpha}$ is independent of $\alpha$. Since $D_{F_{\alpha}}=D_{F_{0}}-\alpha \sum_{i} \partial_{p_{i}}$, while the off diagonal elements of $R^{ \pm}$ are independent of $p_{i}$, Equation (6.4b) follows from Theorem 9, in particular (5.13a), in the case $f(s)=\frac{1}{2} s^{2}$. Hence, by (6.4),

$$
\begin{aligned}
\delta\left(\frac{1}{2} R^{+} R^{-}+\alpha Q\right) & =\frac{1}{2}\left(\delta R^{+}\right) R^{-}+\frac{1}{2} R^{+} \delta R^{-}+\alpha \delta Q \\
& =-\frac{1}{2} \alpha Q R^{-}-\frac{1}{2} \alpha R^{+} Q+\frac{1}{2} \alpha\left(Q R^{-}+R^{+} Q\right)=0
\end{aligned}
$$

and so $\delta W=0$, i.e., $D_{F_{\alpha}} W=[A, W]$. The only thing left to prove, returning to $(x, y)$ coordinates, is that the ( $\left.\operatorname{tr} W^{j}\right)$ 's are in involution, as their algebraic independence is easily seen by considering the case, for all $i$, of $y_{i}$ very large, $x_{i}-x_{i-1}$ very large, and $-x_{i}>0$ very large. As in the proof of Theorem 2 , due to the rational character of the integrals $\operatorname{tr} W^{j}$ in $\left(y_{i}, e^{x_{i}}\right)$, it suffices to prove their involutive character in some open neighborhood. We shall pick the neighborhood in the $(x, y)$ space where for $\alpha>0$, the solutions have the following asymptotic behavior:

$$
\begin{aligned}
& x_{i}=\lambda_{i} t+\beta_{i}+O\left(t^{-1}\right), \quad \lambda_{i}<0, \quad \lambda_{i}<\lambda_{i+1} \text { for all } i, \\
& y_{i}=\lambda_{i} t+O\left(t^{-2}\right), \quad t \rightarrow+\infty .
\end{aligned}
$$

As in Lemma 2.1, such a neighborhood is easily found by converting the differential equations of motion into an integral equation. Then for

$$
Z_{j k}=\delta_{j} y_{j}+\frac{1}{2} i\left(1-\delta_{j k}\right) \operatorname{coth}\left(\frac{1}{2}\left(x_{j}-x_{k}\right)\right),
$$


we have

$$
\begin{aligned}
\lim _{t \rightarrow \infty} Z(t) & =\left\{\lambda_{j} \delta_{j k}+i \sigma_{j k}\right\}, \\
\sigma_{j k} & =-1 \quad \text { if } k>j \\
& =+1 \quad \text { if } k<j \\
& =0 \text { if } k=j,
\end{aligned}
$$

while $\lim _{t \rightarrow \infty} e^{X}=0$. Thus the definition, (6.2), of $W$ implies $\lim _{t \rightarrow \infty} W(t)$ exists and is a function of the $\lambda_{i}$ 's. We then apply the same argument as in Theorem 2 to verify that the $\operatorname{tr} W^{p}, \mathrm{~s}$ are in involution. Also, as in Theorem 2, since the integrals are rational in $\alpha$, the statement of involution for all $\alpha$ follows from the statement for $\alpha>0$.

We now explore this system (6.1) in a sequence of corollaries. Since no collisions can occur we order the particles $x_{i}<x_{i+1}$ for $i=1,2, \ldots, n-1$, all $t$.

Corollary 11.1. All solutions of the system $H_{\alpha}$, for $\alpha>0$, behave asymptotically in the following manner :

$$
\begin{aligned}
& x_{i}= \pm \lambda_{i} t+\beta_{i}^{ \pm}+O\left(t^{-1}\right), \quad t \rightarrow \pm \infty, \quad \lambda_{1}<\lambda_{2}<\ldots<\lambda_{n}<0, \\
& y_{i}= \pm \lambda_{i}+O\left(t^{-2}\right), \\
& \beta_{i}^{+}+\beta_{i}^{-}=2 \log \left(\alpha \lambda_{i}^{2}\right) .
\end{aligned}
$$

In words, the scattering behavior of the system is that of $n$ decoupled particles, each interacting with the origin under the potential $e^{x}$. The effect of the interaction is felt through the strict ordering of the terminal velocities.

Proof. With no loss of generality, assume $\alpha=1$. Then dropping the subscript $H=H_{\alpha}$, for $\alpha=1$ [given by (6.1)], we have

$$
\dot{x}_{i}=\partial H / \partial y_{i}=y_{i}, \quad \ddot{x}_{i}=\dot{y}_{i}=-\partial H / \partial x_{i}, \quad i=1, \ldots, n,
$$

implies

$$
\ddot{x}_{i}=\sum_{j \neq i} \phi\left(x_{i}-x_{j}\right)-e^{x_{i}}, \quad \phi(x)=\frac{1}{4}\left(\operatorname{coth} \frac{1}{2} x\right)\left(\sinh \frac{1}{2} x\right)^{-2} .
$$

We first show that for all $i, \lim _{t \rightarrow \infty} y_{i}(t)$ exists and is nonpositive, for which we use only the following properties of $\phi$ :

$$
\phi^{\prime}(x) \leqq 0, \quad \phi(-x)=-\phi(x), \quad x \phi(x)>0, \quad x \neq 0, \quad \phi(0)=\infty .
$$

From (6.9) we have

$$
\ddot{x}_{1}=\sum_{j>1} \phi\left(x_{1}-x_{j}\right)-e^{x_{1}},
$$

and we note $\phi\left(x_{1}-x_{j}\right),-e^{x_{1}}$ are $<0$, while the energy relation, (6.1), gives an upper bound on $\left|\dot{x}_{i}\right|$. Hence

$$
2(2 H)^{1 / 2} \geqq\left|\int_{-\infty}^{\infty} \ddot{x}_{1} d t\right|=-\int_{-\infty}^{\infty} \ddot{x}_{1} d t=\sum_{j>1} \int_{-\infty}^{\infty} \phi\left(x_{j}-x_{1}\right) d t+\int_{-\infty}^{\infty} e^{x_{1}} d t,
$$

and so $\phi\left(x_{j}-x_{1}\right)>0, e^{x_{1}}$ are integrable on $(-\infty, \infty)$. From this it follows $\dot{x}_{1}(\infty)=\int_{0}^{\infty} x_{1}(t) d t+\dot{x}_{1}(0)$ exists. Now from (6.9) we have $\ddot{x}_{2}+\phi\left(x_{1}-x_{2}\right)$ 
$=\sum_{j>2} \phi\left(x_{2}-x_{j}\right)-e^{x_{2}}$, where again we note $\phi\left(x_{2}-x_{j}\right),-e^{x_{2}}$ are $<0$ for $j>2$. By the previous step and the upper bound on $\left|\dot{x}_{j}\right|$, we have that the left side of the above equation is integrable in $t$ on $(-\infty, \infty)$, and thus so is the right side. Since all terms on the right hand side of the equation are of the same sign, each one of them is integrable, i.e. $\phi\left(x_{2}-x_{j}\right), j \neq 2, e^{x_{2}}$ are thus integrable, and so as before, $\dot{x}_{2}(\infty)$ exists. Inductively we climb our way up to $i=n$, and conclude $\dot{x}_{j}(\infty)$ exists for all $j$, and $e^{x_{j}}$, $\phi\left(x_{i}-x_{j}\right)$ are integrable on $(-\infty, \infty)$ for all $i \neq j$. Setting $\lambda_{j}^{+}=\dot{x}_{j}(\infty)$, since $x_{j}<x_{j+1}$, and $\int_{-\infty}^{\infty} e^{x_{j}(t)} d t$ exists for all $j$, we must have $\lambda_{1}^{+} \leqq \lambda_{1}^{+} \leqq \ldots \leqq \lambda_{n}^{+} \leqq 0$. We now wish to show $\lambda_{1}^{+}<\lambda_{2}^{+}<\ldots<\lambda_{n}^{+}<0$, but first we make some preliminary observations. We compute

$$
8 \dot{\phi}\left(x_{i}-x_{j}\right)=-\left[2 \sinh ^{-2}\left(\frac{1}{2}\left(x_{i}-x_{j}\right)\right)+3 \sinh ^{-4}\left(\frac{1}{2}\left(x_{i}-x_{j}\right)\right)\right]\left(\dot{x}_{i}-\dot{x}_{j}\right),
$$

and so by the energy relation $(6.1), \dot{\phi}\left(x_{i}-x_{j}\right)$ is bounded. We now claim $\lim _{t \rightarrow \infty} \phi\left(x_{i}-x_{j}\right)=0$. To see that, we give without proof the following easy estimate, true for unbounded intervals $I$,

$$
4\left|f^{\prime}\right|_{\infty} \cdot|f|_{1} \geqq|f|_{\infty}^{2}, \quad \text { for } \quad f \in L^{1} \cap L^{\infty},
$$

where

$$
|f|_{1}=\int_{I}|f| d t, \quad|f|_{\infty}=\sup _{t \in I}|f| .
$$

We shall apply (6.10) by picking our interval $I=(t, \infty), f(t)=\phi\left(x_{i}-x_{j}\right)$, and then letting $t \rightarrow \infty$; we see $\left|\phi\left(x_{i}-x_{j}\right)\right|_{\infty}^{2} \rightarrow 0$, as was to be shown. Since $f(t) \rightarrow 0, t \rightarrow \infty$, we must have $\lim _{t \rightarrow \infty} \operatorname{coth}\left(\frac{1}{2}\left(x_{i}-x_{j}\right)\right)= \pm 1$, depending on whether $i>j$ or $i<j$. We are now ready to show $\lambda_{1}^{+}<\lambda_{2}^{+}<\ldots<\bar{\lambda}_{n}^{+}<0$.

First we show $\lambda_{j}<0$. By (6.6), (6.2), and the asymptotic behavior of $\dot{y}_{i}$, $\operatorname{coth}\left(\frac{1}{2}\left(x_{i}-x_{j}\right)\right), e^{x_{1}}$, discussed above, it follows that

$$
\lim _{t \rightarrow \infty} Z^{ \pm}(x(t), y(t)) \equiv T^{ \pm}, \quad \lim _{t \rightarrow \infty} W\left(x(t, y(t))=\frac{1}{2} T^{+} T^{-}\right.
$$

exists, where $T^{ \pm}$equals respectively a lower, upper triangular matrix, with the $\lambda_{j}$ 's in the diagonal, and $\pm i$ respectively in all the lower, upper entries respectively. Hence

$\operatorname{det} \lim _{t \rightarrow \infty} W(t)=\frac{1}{2}\left(\operatorname{det} T^{+}\right)\left(\operatorname{det} T^{-}\right)$

$$
=\frac{1}{2}\left(\prod_{i=1}^{n} \lambda_{i}^{+}\right) \cdot\left(\prod_{i=1}^{n} \lambda_{i}^{+}\right)=\frac{1}{2} \prod_{i=1}^{n}\left(\lambda_{i}^{+}\right)^{2},
$$

but since $W(t)$ undergoes an isospectral deformation in $t$, det $W(0)$ $=\operatorname{det} W(t)=\operatorname{det} W(\infty)$. On the other hand $W=\frac{1}{2}\left(Z^{+}\right)\left(Z^{-}\right)+\alpha e^{x}$, but since $\left(Z^{+}\right)^{*}=Z^{-}$, and as $\alpha>0, W$ is a positive definite Hermitian matrix, which implies $\operatorname{det} W(\infty)=\operatorname{det} W(0)>0$. Thus by our evaluation of $\operatorname{det} W(\infty)$, and since $\lambda_{j}^{+} \leqq 0$, we have shown $\lambda_{j}^{+}<0$ for all $i$.

We now show $\lambda_{i}^{+}<\lambda_{i+1}^{+}$for all $i$, using an argument in [3]. From (6.9) we conclude

$$
e^{x_{n}}-e^{x_{1}}+\left(x_{n}-x_{1}\right)^{*} \geqq 2 \phi\left(x_{n}-x_{1}\right),
$$


and since $0<e^{x_{n}}-e^{x_{n}} \leqq c_{1} e^{\lambda_{n} t}=O\left(t^{-3}\right)$, the function $\psi=x_{n}-x_{1}+c_{2} t^{-1}$ $>x_{n}-x_{1}>0$, with some constant $c_{2}>0$, satisfies

$$
\ddot{\psi} \geqq 2 \phi\left(x_{n}-x_{1}\right) \geqq 2 \phi(\psi)>0 \quad \text { for } t \geqq t_{0},
$$

where we have made use of $\phi^{\prime}(x) \leqq 0, x \phi(x)>0$. Thus $\dot{\psi}$ is monotonic increasing and $\dot{\psi}(\infty)=\lambda_{n}^{+}-\lambda_{1}^{+} \geqq 0$. If $\dot{\psi}(\infty)=0$, then $\dot{\psi}(t)<0$, and since $\psi>0, \psi$ would be bounded. This implies $\phi(\psi)$ is bounded away from zero, which in turn implies $\psi(t)$ is unbounded. This contradiction implies $0<\dot{\psi}(\infty)=\lambda_{n}^{+}-\lambda_{1}^{+}$, and since $\lambda_{1}^{+} \leqq \lambda_{2}^{+} \leqq \ldots \leqq \lambda_{n}^{+}$, there must exist an $s$ such that $\lambda_{s}^{+}<\lambda_{s+1}^{+}$. From this it will follow that $\lambda_{1}^{+}<\lambda_{s}^{+}, \lambda_{s}^{+}<\lambda_{n}^{+}$, and proceeding inductively we can conclude that all the $\lambda_{i}^{+}$'s are different. We show $\lambda_{1}<\lambda_{s}$ assuming $s>1$, the other case being proven in the same way. Since $\lambda_{j}^{+}>\lambda_{s}^{+}, j>s, x_{j}-x_{s} \geqq c_{3} t, c_{3}>0, t \geqq t_{0}, j>s$, we have by (6.9) and $\lambda_{s}^{+}<0$, that

$$
\begin{aligned}
d^{2}\left(x_{s}-x_{1}\right) / d t^{2}= & \sum_{j<s} \phi\left(x_{s}-x_{j}\right)+\sum_{j>1} \phi\left(x_{j}-x_{1}\right)-O\left(t^{-3}\right) \\
& \geqq 2 \phi\left(x_{s}-x_{1}\right)-O\left(t^{-3}\right) .
\end{aligned}
$$

Thus we are in precisely the same position with $x_{s}-x_{1}$ as we were in before with $x_{n}-x_{1}$, and so conclude $\lambda_{s}^{+}>\lambda_{1}^{+}$. We have thus shown $\lambda_{1}^{+}<\lambda_{2}^{+} \ldots<\lambda_{n}^{+}<0$ and similarly for $t \rightarrow-\infty$, i.e., if $\dot{x}_{i}(-\infty)=\lambda_{i}^{-}$, we find $\lambda_{1}^{-}>\lambda_{2}^{-}>\ldots<\lambda_{n}^{-}>0$.

Using arguments similar to those of Lemma 2.1 , we easily show

$$
\begin{aligned}
x_{i}(t) & =\lambda_{i}^{ \pm} t+\beta_{i}^{ \pm}+O\left(t^{-1}\right), \quad t \rightarrow \pm \infty, \text { for all } i \\
y_{i} & =\lambda_{i}^{ \pm}+O\left(t^{-2}\right),
\end{aligned}
$$

and so we must prove $\lambda_{i}^{+}=-\lambda_{i}^{-}$. We first prove this fact for all $\left|\lambda_{i}^{+}\right|$very large. In that case by (6.11), the spectrum of $2 W$ is 'relatively' close to $\left(\lambda_{1}^{+}\right)^{2},\left(\lambda_{2}^{+}\right)^{2}, \ldots\left(\lambda_{n}^{2}\right)^{2}$, and by the implicit function theorem there exists a $C^{\prime}$ diffeomorphism $\tau$, completely determined by $(6.11)$ and defined by $\tau\left(\left(\lambda_{1}^{+}\right)^{2},\left(\lambda_{2}^{+}\right)^{2}, \ldots,\left(\lambda_{n}^{+}\right)^{2}\right)=\left(\varrho_{1}, \varrho_{2}, \ldots, \varrho_{n}\right)$, $\varrho_{1}>\varrho_{2}>, \ldots,>\varrho_{n}$, where $\left(\varrho_{1}, \varrho_{2}, \ldots, \varrho_{n}\right)$ equals the spectrum of $W$. For the domain $N$ of $\tau$, we fix a point defined by $\left(\lambda_{i}^{+}\right)^{2}=v_{i}=(n-i+1) M, i=1, \ldots, n, M$ sufficiently large and positive, and let $N=\left\{\left(\left(\lambda_{1}^{+}\right)^{2}, \ldots,\left(\lambda_{n}^{+}\right)^{2}\right)||\left(\lambda_{i}^{+}\right)^{2}-v_{i} \mid<\mu(M)\right\}, \mu(M)<1$, and being picked sufficiently small to ensure $\tau$ is a diffeomorphism. On the other hand, in the above neighborhood, since the spectrum of $W$ is very large, by $(6.11)$ in the case of $\lambda^{-}$, we know that the $\left(\lambda_{i}^{-}\right)^{2}, i=1, \ldots, n$, must be very large and "relatively" close to the spectrum of $W$. In fact, using the implicit function theorem once again, and (6.11), in the case of $\lambda^{-}$, noting that the form of (6.11) guarantees that the spectrum of $W$ is unchanged upon the inversion $\lambda_{i}^{+} \rightarrow-\lambda_{i}^{+}, \mathrm{i}=1,2, \ldots, n$, we must have $\tau\left(\left(\lambda_{1}^{-}\right)^{2},\left(\lambda_{2}^{-}\right)^{2}, \ldots,\left(\lambda_{n}^{-}\right)^{2}\right)=\left(\varrho_{1}, \ldots, \varrho_{n}\right)$. While by the uniqueness clause in the implicit function theorem and (6.11), this implies $\left(\lambda_{i}^{+}\right)^{2}=\left(\lambda_{i}^{-}\right)^{2}$, and hence $\lambda_{i}^{+}=-\lambda_{i}^{-}$.

We now wish to prove $\lambda_{i}^{+}=-\lambda_{i}^{-}$for all $(x, y) \in \Omega$. For that we observe that $(6.12)$, as in Lemma 2.1, enables you to construct a canonical diffeomorphism of $\Omega, \phi_{+}$, defined by

$$
\phi_{+}(x, y)=\left(\lambda^{+}, \beta^{+}\right), \quad \lambda^{+}=\left(\lambda_{1}^{+}, \ldots, \lambda_{n}^{+}\right), \quad \beta=\left(\beta_{1}^{+}, \ldots, \beta_{n}^{+}\right),
$$


and similarly one defines $\phi_{-}(x, y)=\left(\lambda^{-}, \beta^{-}\right)$. We can then define $\phi=\phi_{+}{ }^{\circ} \phi_{-}^{-1}$, a canonical diffeomorphism of $\Omega$, by $\phi\left(\lambda^{-}, \beta^{-}\right)=\left(\lambda^{+}, \beta^{+}\right)$, which, in particular, is continuous. Now by (6.11),

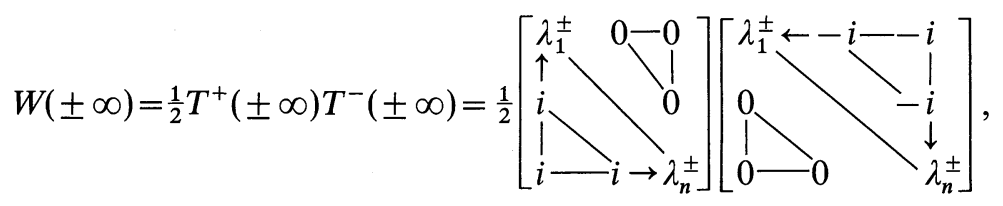

and so the coefficients $a_{k}$ of the characteristic polynomial, $\operatorname{det}(z I-W( \pm \infty))$ $=\sum_{k=1}^{n} z^{k} a_{k}\left(\lambda^{ \pm}\right)$depend on $\lambda_{j}^{+}$or $\lambda_{j}^{-}$only. Thus since $W(+\infty) \sim W(-\infty)$, the map $\lambda^{-} \rightarrow \lambda^{+}$is given by the $n$ algebraically independent relations $a_{k}\left(\lambda^{-}\right)=a_{k}\left(\lambda^{+}\right)$, $k=1, \ldots, n$. On the other hand, we have shown $-\lambda_{k}^{-}=\lambda_{k}^{+}$, for all $k$ in some neighborhood, and thus by the continuity of $\phi$, and the identity theorem for analytic functions, $-\lambda_{k}^{-}=\lambda_{k}^{+}, k=1, \ldots, n$, for all $\Omega$.

To prove (6.8), we observe once again, applying an argument of Moser's [15], that the maps $(x, y) \rightarrow\left(\beta^{ \pm}, \lambda^{ \pm}\right)$are canonical, and hence

$$
\sum_{i=1}^{n} d \beta_{i}^{+} \wedge d \lambda_{i}^{+}=\sum_{i=1}^{n} d x_{i} \wedge d y_{i}=\sum_{i=1}^{n} d \beta_{i}^{-} \wedge d \lambda_{i}^{-}
$$

which implies, along with the self explanatory definition of $u$,

$$
d u=d \sum_{i}\left(\beta_{i}^{+}+\beta_{i}^{-}\right) d \lambda_{i}=0, \quad \lambda_{i}=\lambda_{i}^{+}=-\lambda_{i}^{-} .
$$

By the Poincaré Lemma for the convex domain $\Omega$, we find $u=d S(\lambda)$, and so

$$
\beta_{i}^{+}+\beta_{i}^{-}=\partial S(\lambda) / \partial \lambda_{i}
$$

We first show $S=\sum_{i=1}^{n} \tilde{S}\left(\lambda_{i}\right)$, and then we evaluate $S$ explicitly. To see $\tilde{S}$ has the above decomposition, we note that the one particle system with

$$
H(x, y)=\frac{1}{2} y^{2}+e^{x},
$$

has for its most general solution

$$
x=\log \left[2 \lambda^{2} \operatorname{sech}^{2}(\lambda t+\delta)\right], \quad \lambda>0,
$$

and hence

$$
x(t)=\mp 2 \lambda t+\beta^{ \pm}+O\left(t^{-1}\right), \quad t \rightarrow \pm \infty,
$$

where $\beta^{ \pm}=\left(\log \left(2 \lambda^{2}\right) \mp 2 \delta+2 \log 2\right)$. Thus if we have the $n$-particle system with

$$
H=\sum_{i=1}^{n}\left(\frac{1}{2} y_{i}^{2}+e^{x_{i}}\right),
$$

we would have

$$
x_{j}-x_{i}=2 \log \left[\frac{\lambda_{j} \cosh \left(\lambda_{i} t+\delta_{i}\right)}{\lambda_{i} \cosh \left(\lambda_{j} t+\delta_{j}\right)}\right],
$$


where the notation obviously comes from (6.14). Now fixing $\lambda_{1}>\lambda_{2}>\ldots>\lambda_{n}>0$, if $\delta_{i-1}-\delta_{i}=m>0$, sufficiently large, for all $i$, we conclude from (6.17) that $x_{i+1}(t)>x_{i}(t)$ for all $i, t$. If we let $m \rightarrow \infty$, or what comes to the same thing $\beta_{i+1}^{+}-\beta_{i}^{+} \rightarrow \infty$ for all $i$ then $\inf _{i, t}\left(x_{i+1}-x_{i}\right) \rightarrow \infty$. We are now in a position to perform a scattering experiment with the system $H_{\alpha}$. Namely, to evaluate $S$, since it only depends on the terminal velocities, we may choose a set of scattering data for a fixed set of final velocities, i.e.,

$$
x_{i}(t)=-2 \lambda_{i} t+\beta_{i}^{+}+O\left(t^{-1}\right), \quad t \rightarrow \infty,
$$

where $\beta_{i+1}^{+}-\beta_{i}^{+}=m, m$ large. Then by the above considerations, the system $H_{\alpha}$ decouples as $m \rightarrow \infty$, hence

$$
\partial S / \partial \lambda_{i}=\beta_{i}^{+}+\beta_{i}^{-}=2 \log \left(2 \lambda_{i}\right)^{2}+O\left(m^{-1}\right) .
$$

But since $\left(\partial / \partial \lambda_{i}\right) S\left(\lambda_{1}, \ldots, \lambda_{n}\right)$ does not depend on $m$, we must have $\beta_{i}^{+}+\beta_{i}^{-}$ $=2 \log \left(2 \lambda_{i}\right)^{2}$. Lifting the requirement that $\alpha=1$, and returning to the notation of the theorem, we find $\beta_{i}^{+}+\beta_{i}^{-}=2 \log \left(\alpha \lambda_{i}^{2}\right)$.

Corollary 11.2. The explicit solution of the equations of motion for $\alpha \neq 0$ are given by

$$
e^{X} \sim\left(2 \alpha^{-1} \dot{p} p^{-1}\right)^{\circ}, \quad p=A_{1} e^{\Lambda t} A_{2}+A_{3} e^{-\Lambda t} A_{4},
$$

where $\sim$ indicates similarity equivalence. Here the $A_{i}$ 's are constant matrices described in the proof, and in case $\alpha>0$, we have

$$
2 \Lambda=\operatorname{diag}\left(-\lambda_{1},-\lambda_{2}, \ldots,-\lambda_{n}\right),
$$

with $\lambda_{i}$ being the same as in (6.7). Thus the symmetric polynomials of $e^{x_{i}}$, for $\alpha>0$, are rational in $e^{ \pm 1 / 2 \lambda_{j} t}, j=1, \ldots, n$. Moreover, if we define the $2 n \times 2 n$ matrices $J, \hat{K}$ by

$$
J=\left[\begin{array}{cc}
0, & I \\
\frac{1}{2} W, & \frac{1}{2} C
\end{array}\right], \quad \hat{K}=\left[\begin{array}{cc}
K, & 0 \\
0, K
\end{array}\right],
$$

where $C_{j k}=i\left(1-\delta_{j k}\right)$, and $W(x, y), K(x)$ as defined in (6.2), (6.3), then

$$
D_{H_{\alpha}} J=[\tilde{K}, J] \text {, }
$$

i.e., $J$ is isospectral. For $\alpha>0$, the spectrum of $J$ is $\pm \frac{1}{2} \lambda_{i}, i=1, \ldots, n$, but in any case, the spectrum occurs in \pm pairs. Finally $\Lambda$ satisfies the quadratic matrix equations

$$
\tilde{W} \pm \tilde{C}=2 \Lambda^{2},
$$

where

$$
\tilde{W}=V_{ \pm}^{-1} W V_{ \pm}, \quad \tilde{C}=V_{ \pm}^{-1} C V_{ \pm},
$$

respectively, for some nonsingular $n$ by $n$ matrices $V_{ \pm}$.

Proof. We first compute the time dependence of $e^{X}$, i.e. (6.19), and for that we go over to $\left(q_{1}, \ldots, q_{n}, p_{1}, \ldots, p_{n}\right)$ coordinates, as in the proof of Theorem 11, using the same notation as in that proof. We define the matrices $q, r^{ \pm}$by

$$
q=U^{-1} Q U, \quad r^{ \pm}=U^{-1} R^{ \pm} U,
$$


where $U$ is a matrix satisfying $U(0)=I, \dot{U}=A U, A$ as in (6.3). This matrix $q$ is not to be confused with the coordinates $\left(q_{1}, \ldots, q_{n}\right)$. The Equations (6.4) are respectively equivalent to (see Remark 1 of Section 2),

$$
\left\{\begin{array}{c}
\dot{q}=q r^{-}=r^{+} q, \text { since } q r^{-}=r^{+} q, \\
\dot{r}^{ \pm}=-\alpha q .
\end{array}\right\}
$$

Since $R^{+}-R^{-}=C,[C, A]=0, \dot{U}=A U$, it follows that $U^{-1} C U=C$, and so we have

$$
r^{+}-r^{-}=C
$$

That $W$ is isospectral is equivalent to (see Remark 1 of Section 2)

$$
\frac{1}{2} r^{+} r^{-}+\alpha q=W_{0},
$$

where the subscript shall now, and for the remainder of this section, indicate evaluation at $t=0$. By the above we have

$$
\begin{aligned}
& \frac{1}{2}\left(r^{-}+C\right) r^{-}-\dot{r}^{-}=W_{0}, \text { i.e. } \\
& \frac{1}{2}\left(r^{-}\right)^{2}-\dot{r}^{-}=W_{0}-\frac{1}{2} C r^{-} .
\end{aligned}
$$

Defining $p$ as the solution of the linear differential equation,

$$
r^{-}=-2 \dot{p} p^{-1}, \quad p(0)=I, \quad\left[\text { hence } \dot{p}(0)=-\frac{1}{2} r_{0}^{-}=-\frac{1}{2} Z_{0}^{-}\right],
$$

and substituting this into (6.26), we compute

$$
\frac{1}{2}\left(-2 \dot{p} p^{-1}\right)\left(-2 \dot{p} p^{-1}\right)-\left(-2 \ddot{p} p^{-1}+2 \dot{p} p^{-1} \dot{p} p^{-1}\right)=W_{0}-\frac{1}{2} C\left(-2 \dot{p} p^{-1}\right),
$$

which implies

$$
\ddot{p}=\frac{1}{2} W_{0} p+\frac{1}{2} C \dot{p}
$$

Defining the $n$ by $2 n$ matrix $P=\left(\begin{array}{c}p \\ \dot{p}\end{array}\right)$, we conclude

$$
\dot{P}=J_{0} P \text {, hence } P=e^{J_{0} t} P_{0},
$$

with $J$ defined in the statement of this corollary.

Recalling $Q=\operatorname{diag}\left(q_{1}, \ldots, q_{n}\right)=\operatorname{diag}\left(e^{x_{1}}, \ldots, e^{x_{n}}\right)=e^{X}$, see $(6.3)$, we compute, using (6.24), (6.25), and (6.27),

$$
\begin{aligned}
U^{-1} e^{X} U & =U^{-1} Q U=q=-\alpha^{-1} \dot{r}^{-} \\
& =-\alpha^{-1}\left(-2 \dot{p} p^{-1}\right)^{\cdot}=2 \alpha^{-1}\left(\dot{p} p^{-1}\right)^{\cdot}
\end{aligned}
$$

hence

$$
U^{-1} e^{X} U=2 \alpha^{-1}\left(\dot{p} p^{-1}\right)^{\cdot} .
$$

Assuming for the moment the statement of the corollary concerning the spectrum of $J$, we may write, using (6.28),

$$
\left(\begin{array}{l}
p \\
\dot{p}
\end{array}\right)=P=e^{J_{0} t} P_{0}=E e^{\underline{\Lambda} t}\left(E^{-1} P_{0}\right),
$$


with $\underline{\Lambda}=\operatorname{diag}(\Lambda,-\Lambda), \Lambda$ an $n \times n$ matrix which takes, for $\alpha>0$, the form given in (6.20), and $E$ a matrix which block diagonalizes $J_{0}$. This expression for $P$, in conjunction with the above expression for $U e^{X} U^{-1}$, yields (6.19).

We now analyze the spectrum of $J$, but first we must verify (6.22), i.e. $D_{H_{\alpha}} J=[\tilde{K}, J]$. We compute

$$
D_{H_{\alpha}} J=\left[\begin{array}{cc}
0, & 0 \\
\frac{1}{2} D_{H_{\alpha}} W, & 0
\end{array}\right],
$$

while by (6.21),

$$
[\hat{K}, J]=\frac{1}{2}\left[\begin{array}{cc}
0, & 0 \\
{[K, W],} & {[K, C]}
\end{array}\right]=\frac{1}{2}\left[\begin{array}{cc}
0, & 0 \\
{[K, W],} & 0
\end{array}\right],
$$

and thus by Theorem 11, (6.22) is verified,

We now assume $\alpha>0$, returning to $(x, y)$ coordinates, and proceed to prove that the spectrum of $J$ (which by (6.22) is constant along an orbit) is $\pm \frac{1}{2} \lambda_{i}, i, \ldots, n$. Now, since $J(t)$ is an isospectral matrix, it is sufficient to prove

$$
J(\infty)=\lim _{t \rightarrow \infty} J(t)=\left[\begin{array}{cc}
0, & I \\
\frac{1}{4} T^{+} T^{-}, & \frac{1}{2} C
\end{array}\right]
$$

[see (6.13)], has the above spectrum. Defining the matrices $\Delta, \lambda$, by

$$
\Delta_{j k}=\left\{\begin{array}{lll}
i & \text { if } & k<j \\
0 & \text { if } & k \geqq j
\end{array}\right\}, \quad \lambda=\operatorname{diag}\left(\lambda_{1}, \lambda_{2}, \ldots, \lambda_{n}\right),
$$

(6.13) implies

$$
T^{+} T^{-}=(\lambda+\Delta)\left(\lambda+\Delta^{*}\right), \quad C=\Delta-\Delta^{*} .
$$

Thus to prove that the spectrum of $J(\infty)$ is $\pm \frac{1}{2} \lambda_{i}, i=1, \ldots, n$, it is sufficient to prove the seemingly stronger, purely algebraic statement:

Lemma 11. 3. If

$$
M_{\varepsilon}(\lambda)=\left[\begin{array}{cc}
0 & , \\
\frac{1}{4}(\lambda+\varepsilon \Delta)\left(\lambda+\varepsilon \Delta^{*}\right), & \frac{1}{2} \varepsilon\left(\Delta-\Delta^{*}\right)
\end{array}\right],
$$

where $\lambda=\operatorname{diag}\left(\lambda_{1}, \ldots, \lambda_{n}\right)$, the spectrum of $M_{\varepsilon}(\lambda)$ is independent of $\varepsilon$, and in particular the spectrum of $M_{0}(\lambda)$ agrees with the spectrum of $M_{1}(\lambda)=J(\infty)$.

Proof. For the proof, which need only be given for the case $\lambda_{1}<\lambda_{2}<\ldots<\lambda_{n}<0$, we employ the asymptotic description of the orbits of $H_{\alpha}$, formula (6.7). First we make some preliminary observations. Defining

$$
\lambda=\lambda(\varepsilon)=\varepsilon^{-1} \lambda^{\prime}, \quad B=\left[\begin{array}{cc}
I, & 0 \\
0, & \varepsilon I
\end{array}\right],
$$

we compute

$$
\varepsilon B M_{1}(\lambda) B^{-1}=M_{\varepsilon}\left(\lambda^{\prime}\right), \quad \text { i.e., } \quad \varepsilon M_{1}(\lambda) \sim M_{\varepsilon}\left(\lambda^{\prime}\right) .
$$


For the rest of the proof of Lemma 11.3, we fix $\lambda^{\prime}, \lambda_{1}^{\prime}<\lambda_{2}^{\prime}<\ldots<\lambda_{n}^{\prime}<0$. We then pick $\delta_{1}=\delta_{1}\left(\lambda^{\prime}\right)$ so that

$$
\left|M_{\varepsilon}\left(\lambda^{\prime}\right)-M_{0}\left(\lambda^{\prime}\right)\right|<\frac{1}{2} \delta_{2} \text {, if }|\varepsilon|<\delta_{1}<\delta_{2}<1,
$$

with $\delta_{2}=\delta_{2}\left(\lambda^{\prime}\right)$ to be determined in the course of the discussion, and $|A|=\sup _{i=1, \ldots, n}\left(\sum_{j=1}^{n}\left|A_{i j}\right|\right)$. We now fix $\varepsilon$ for the remainder of this lemma, $\varepsilon$ only having the property $|\varepsilon|<\delta_{1}$.

Observe that for any matrix of the form $M_{0}\left(\lambda^{\prime}\right)+S, S$ a small matrix with $|S|<\delta_{2}$, we may find a matrix $D$ such that

$$
\left\{\begin{array}{l}
D^{-1}\left(M_{0}\left(\lambda^{\prime}\right)+S\right) D=\operatorname{diag}\left(\mu_{1}^{+}, \ldots, \mu_{n}^{+}, \mu_{1}^{-}, \ldots, \mu_{n}^{-}\right) \\
=\left[\begin{array}{cc}
\mu^{+}, & 0 \\
0, & \mu^{-}
\end{array}\right], \quad \mu_{i}^{ \pm}=\frac{1}{2}\left(\mp \lambda_{i}^{\prime}+O\left(\delta_{2}\right)\right), \quad i=1, \ldots, n
\end{array}\right\} .
$$

In addition, $D$ may be taken to be of the form

$$
\left\{\begin{array}{l}
D=\left[\begin{array}{ll}
d_{11}, & d_{12} \\
d_{21}, & d_{22}
\end{array}\right]=\left[\begin{array}{cc}
I, & I \\
-\frac{1}{2} \lambda^{\prime}, & \frac{1}{2} \lambda^{\prime}
\end{array}\right]+O\left(\delta_{2}\right), \\
\text { and so } \\
D^{-1}=\left[\begin{array}{rr}
\frac{1}{2} I, & -\left(\lambda^{\prime}\right)^{-1} \\
\frac{1}{2} I, & \left(\lambda^{\prime}\right)^{-1}
\end{array}\right]+O\left(\delta_{2}\right)
\end{array}\right\}
$$

Note that all the $O$ 's which appear now, and for the rest of the discussion, depend only on $\lambda^{\prime}$.

We proceed with the proof of Lemma 11.3, having fixed $\lambda^{\prime}$, and then $\varepsilon$, and therefore $\lambda=\varepsilon^{-1} \lambda^{\prime}$, as previously discussed. We first consider a particular orbit with asymptotic behavior given by (6.7), and any choice of $\beta=\left(\beta_{1}, \ldots, \beta_{n}\right)$. We then pick the origin of the orbit, i.e., replacing $t$ by $t+\bar{t}$, with $\bar{t}$ chosen so large that

$$
\left\{\begin{array}{cc}
J_{0}(x(t), y(t))=M_{1}(\lambda)+S_{1}, & \left|S_{1}\right|<\frac{1}{2} \delta_{2}, \\
Z_{0}^{-}(x(t), y(t))=\left(\lambda+\Delta^{*}\right)+S_{2}, & \left|S_{2}\right|<1
\end{array}\right\} .
$$

Since by (6.7),

$$
\lim _{t \rightarrow \infty} J(x(t), y(t))=M_{1}(\lambda), \quad \lim _{t \rightarrow \infty} Z^{-}(x(t), y(t))=\lambda+\Delta^{*},
$$

such a $\bar{t}$ can always be chosen.

With the above chosen orbit, we rescale time, $t \rightarrow \varepsilon^{-1} t=t^{\prime}$, and so in (6.7), the terminal velocities $\lambda$ are replaced by $\varepsilon \lambda=\lambda^{\prime}$. In what follows, we shall indicate the transformed quantities by primed letters. We compute

$$
\left(\begin{array}{l}
p \\
\dot{p}
\end{array}\right)=P \rightarrow B P=P^{\prime}=\left(\begin{array}{l}
p^{\prime} \\
\dot{p}^{\prime}
\end{array}\right), \quad B=\left[\begin{array}{cc}
I, & 0 \\
0, & \varepsilon I
\end{array}\right],
$$

while

$$
\dot{P}=J_{0} P \rightarrow \varepsilon B \dot{P}=\left(\varepsilon B J_{0} B^{-1}\right)(B P),
$$


which may be conveniently written as

$$
d P^{\prime} / d t^{\prime}=J_{0}^{\prime} P^{\prime}, \quad J_{0}^{\prime}=\varepsilon B J_{0} B^{-1} .
$$

Using (6.35), (6.31), we compute

$$
\begin{aligned}
J_{0}^{\prime} & =\varepsilon B J_{0} B^{-1}=\varepsilon B\left(M_{1}(\lambda)+S_{1}\right) B^{-1} \\
& =M_{\varepsilon}\left(\lambda^{\prime}\right)+\varepsilon B S_{1} B^{-1}=M_{0}\left(\lambda^{\prime}\right)+S_{3}+S_{4},
\end{aligned}
$$

with

$$
S_{3}=M_{\varepsilon}\left(\lambda^{\prime}\right)-M_{0}\left(\lambda^{\prime}\right), \quad S_{4}=\varepsilon B S_{1} B^{-1} .
$$

Since by (6.32), $|\varepsilon|<1,\left|S_{4}\right| \leqq|B| \cdot\left|S_{1}\right| \cdot\left|\varepsilon B^{-1}\right| \leqq\left|S_{1}\right|<\frac{1}{2} \delta_{2}$, and so, again by (6.32), we find

$$
J_{0}^{\prime}=M_{0}\left(\lambda^{\prime}\right)+S, \quad|S|<\delta_{2} .
$$

We are now in a position to use (6.33), as (6.37) implies, with $D$ defined in (6.33),

$$
P^{\prime}=e^{J_{0} t} P_{0}^{\prime}=D\left\{\exp \left[\left(D^{-1} J_{0}^{\prime} D\right) t\right]\right\}\left(D^{-1} P_{0}^{\prime}\right),
$$

and so by (6.38) applied to (6.33), (6.34), we find

$$
P^{\prime}=\left[\begin{array}{ll}
d_{11}, & d_{12} \\
d_{21}, & d_{22}
\end{array}\right] \cdot\left[\begin{array}{cc}
e^{\mu^{+} t}, & 0 \\
0, & e^{\mu^{-} t}
\end{array}\right] \cdot\left[\begin{array}{l}
p_{1} \\
p_{2}
\end{array}\right], \quad\left[\begin{array}{l}
p_{1} \\
p_{2}
\end{array}\right]=D^{-1} P_{0}^{\prime},
$$

and so conclude

$$
p^{\prime}=d_{11} e^{\mu^{+} t} p_{1}+d_{12} e^{\mu^{-t}} p_{2} .
$$

We shall now come to the determination of $\delta_{2}=\delta_{2}\left(\lambda^{\prime}\right)$, and then we will finish the proof of this lemma. By (6.36), (6.27),

$$
p_{0}^{\prime}=B P_{0}=\left[\begin{array}{cc}
I, & 0 \\
0, & \varepsilon I
\end{array}\right]\left[\begin{array}{c}
I \\
-\frac{1}{2} Z_{0}^{-}
\end{array}\right]=\left[\begin{array}{c}
I \\
-\frac{1}{2} \varepsilon Z_{0}^{-}
\end{array}\right],
$$

and so by (6.39), (6.34), we find

$$
p_{1}=\left(\frac{1}{2} I\right)(I)+\left(-\lambda^{\prime}\right)^{-1}\left(-\frac{1}{2} \varepsilon Z_{0}^{-}\right)+O\left(\delta_{2}\right)\left(I-\frac{1}{2} \varepsilon Z_{0}^{-}\right) .
$$

From (6.35) we have

$$
\left(-\lambda^{\prime}\right)^{-1}\left(-\frac{1}{2} \varepsilon Z_{0}^{-}\right)=\frac{1}{2} \lambda^{-1} Z_{0}^{-}=\frac{1}{2} I+\frac{1}{2} \varepsilon\left(\lambda^{\prime}\right)^{-1}\left(\Delta^{*}+S_{2}\right),
$$

while

$$
I-\frac{1}{2} \varepsilon Z_{0}^{-}=I-\frac{1}{2} \varepsilon\left(\lambda+\Delta^{*}+S_{2}\right)=I-\frac{1}{2} \lambda^{\prime}-\frac{1}{2} \varepsilon \Delta^{*}-\frac{1}{2} \varepsilon S_{2} .
$$

Since by (6.32), (6.35), $|\varepsilon|<\delta_{2},\left|S_{2}\right|<1$, we conclude from the above

$$
\begin{aligned}
p_{1} & =I+S_{5}, \quad \text { with } \quad\left|S_{5}\right|<\sigma\left(\delta_{2}\right), \\
\sigma\left(\delta_{2}\right) & =\frac{1}{2}\left|\lambda_{n}^{\prime}\right|^{-1} \delta_{2}(n+1)+O\left(\delta_{2}\right)\left(1+\frac{1}{2}\left|\lambda_{1}^{\prime}\right|+\frac{1}{2} \delta_{2}(n+1)\right) .
\end{aligned}
$$

We now choose $\delta_{2}=\delta_{2}\left(\lambda^{\prime}\right)$, so that $0<\delta_{2}<1, \sigma\left(\delta_{2}\right)<1$, and in addition $\delta_{2}$ is in the domain of validity of the estimates in (6.33), (6.34). The remark concerning the $\lambda^{\prime}$ dependence of $O$ ensures $\delta_{2}=\delta_{2}\left(\lambda^{\prime}\right)$. 
We now compute the positive spectrum of $J_{0}^{\prime}$. Recalling $q=U^{-1} e^{X} U$, [see (6.29)], and substituting $t \rightarrow t^{\prime}, \lambda \rightarrow \lambda^{\prime}$, in the asymptotic description of the orbit, (6.7), we conclude, using (6.24)

$$
\begin{aligned}
U(t) & =U(\infty)+O\left(\left(t^{\prime}\right)^{-1}\right), \quad \lim _{t^{\prime} \rightarrow \infty} U\left(t^{\prime}\right)=U(\infty), \\
q_{i} & =e^{x_{i}}=e^{\beta_{i}} e^{\lambda_{i} t^{\prime}}\left(1+O\left(\left(t^{\prime}\right)^{-1}\right)\right) \\
\dot{q}_{i} & =y_{i} e^{x_{i}}=\lambda_{i}^{\prime} e^{\beta_{i}} e^{\lambda_{i} t^{\prime}}\left(1+O\left(\left(t^{\prime}\right)^{-1}\right)\right. \\
i & =1, \ldots, n, \text { and so } \\
q^{-1} \dot{q} & =\left[U^{-1}(\infty)\left(\operatorname{diag}\left(\lambda_{1}^{\prime}, \ldots, \lambda_{n}^{\prime}\right)\right) \mathrm{U}(\infty)\right] \cdot\left(1+O\left(\left(t^{\prime}\right)^{-1}\right)\right),
\end{aligned}
$$

where these $O$ 's depend on our fixed initial data. On the other hand, using (6.40), and the nonsingularity of the matrices $d_{11}, p_{1}$, implicit in (6.34), (6.41) respectively, and since by (6.25), (6.27), $q^{-1} \dot{q}=r^{-}=-2 \dot{p} p^{-1}$, we conclude

$$
\lim _{t^{\prime} \rightarrow \infty} q^{-1} \dot{q}=\lim _{t^{\prime} \rightarrow \infty}\left(-2 \dot{p} p^{-1}\right)=-2 d_{11} \mu^{+} d_{11}^{-1} .
$$

This expression coupled with (6.42) yields

$$
U^{-1}(\infty)\left(\operatorname{diag}\left(\lambda_{1}^{i}, \ldots, \lambda_{n}^{\prime}\right)\right) U(\infty)=-2 d_{11} \mu^{+} d_{11}^{-1} .
$$

We thus find that the positive eigenvalues of $J_{0}^{\prime}, \mu_{i}^{+}=-\frac{1}{2} \lambda_{i}^{\prime}, i=1, \ldots, n$, with no error term $O\left(\delta_{2}\right)$, as in (6.33). Thus $\mu_{i}^{+}, i=1, \ldots, n$, is contained in the spectrum of [see 6.37],

$$
J_{0}^{\prime}=\varepsilon B J_{0} B^{-1} \sim \varepsilon B J(\infty) B^{-1}, \quad J(\infty)=\lim _{t \rightarrow \infty} J(x(t), y(t)),
$$

where $\sim$ denotes similarity equivalence, since $J(x(t), y(t))$ undergoes an isospectral deformation by (6.22). By (6.7), $J(\infty)=M_{1}(\lambda)$, and thus by (6.31),

$$
J_{0}^{\prime} \sim \varepsilon B M_{1}(\lambda) B^{-1}=M_{\varepsilon}\left(\lambda^{\prime}\right) .
$$

We have thus shown for arbitrary $\varepsilon$ satisfying $|\varepsilon|<\delta_{1}=\delta_{1}\left(\lambda^{\prime}\right)$, that the positive spectrum of $M_{\varepsilon}\left(\lambda^{\prime}\right)$ contains $-\frac{1}{2} \lambda_{i}^{\prime}, i=1, \ldots, n$. By the same argument for $t^{\prime} \rightarrow-\infty$, after having first shifted our origin appropriately, we also conclude that $\frac{1}{2} \lambda_{i}^{\prime}$, $i=1, \ldots, n$ is contained in the spectrum of $M_{\varepsilon}\left(\lambda^{\prime}\right)$. Hence, $M_{\varepsilon}\left(\lambda^{\prime}\right)$ has full spectrum $\pm \frac{1}{2} \lambda_{i}^{\prime}, i=1, \ldots, n$, at least for $|\varepsilon|<\delta_{1}=\delta_{1}\left(\lambda^{\prime}\right)$. We therefore have proven Lemma 11.3 for $\varepsilon$ small, but since $M_{\varepsilon}(\lambda)$ is a polynomial in $\varepsilon$, the proof is finished.

To complete the proof of Lemma 11.2, we must verify (6.23). To accomplish this end, we shall construct a meromorphic invertible matrix function of $(x, y)$, which diagonalizes $J$, namely

$$
E=E(x, y)=\left[\begin{array}{ll}
e_{11}, & e_{12} \\
e_{21}, & e_{22}
\end{array}\right],
$$

possessing the property that $e_{i j}, i, j=1,2$, is invertible. Assuming for the moment the construction of such an $E$, by (6.21), (6.20), and the spectral resolution of $J$, we have

$$
\left[\begin{array}{cc}
0, & I \\
\frac{1}{2} W, & \frac{1}{2} C
\end{array}\right]\left[\begin{array}{ll}
e_{11}, & e_{12} \\
e_{21}, & e_{22}
\end{array}\right]=\left[\begin{array}{ll}
e_{11}, & e_{12} \\
e_{21}, & e_{22}
\end{array}\right]\left[\begin{array}{cc}
\Lambda, & 0 \\
0, & -\Lambda
\end{array}\right],
$$


which implies

$$
\left\{\begin{array}{c}
e_{21}=e_{11} \Lambda \quad, \quad \begin{array}{c}
e_{22}=-e_{12} \Lambda \\
\frac{1}{2} W e_{11}+\frac{1}{2} C e_{21}=e_{21} \Lambda,
\end{array} \quad \frac{1}{2} W e_{12}+\frac{1}{2} C e_{22}=-e_{22} \Lambda
\end{array}\right\},
$$

from which we conclude

$$
\frac{1}{2} W e_{11}+\frac{1}{2} C e_{11} \Lambda=e_{11} \Lambda^{2}, \quad \frac{1}{2} W e_{12}-\frac{1}{2} C e_{12} \Lambda=e_{12} \Lambda^{2},
$$

which clearly implies (6.23), with $V_{+}=e_{11}, V_{-}=e_{12}$. Note by (6.45) and the nonsingularity of $\Lambda$, that once we have constructed $E(x, y)$ satisfying $(6.44)$, the invertibility of $e_{11}, e_{12}$ implies the invertibility of $e_{i j}, i, j=1,2$.

First we shall construct $E(x, y)$ satisfying (6.44), and then proceed to show the invertibility of its blocks. The construction shall proceed in two steps, i.e., we shall take $E$ of the form $E_{1}(x, y) E_{2}(\lambda)$. To construct $E_{1}$, we first observe that using the arguments of Remark 2 of Section 2 , we may algebraically construct a global unitary matrix $\tilde{U}(x, y)$ such that

$$
\tilde{U}^{-1}(x, y) W(x, y) \tilde{U}(x, y)=\frac{1}{4}(\lambda+\Delta)\left(\lambda+\Delta^{*}\right), \quad \tilde{C} \tilde{U}=\tilde{U}^{*} \tilde{C}=\tilde{C},
$$

$\tilde{C}=C+i I$, with $\lambda, \Delta$ defined as in Lemma $11.3, \frac{1}{2}(\lambda+\Delta)\left(\lambda+\Delta^{*}\right)$ being the limiting value of $W(x, y)$ along an orbit with initial data $(x, y)$. It follows by $(6.21),(6.46)$, that if we define the $2 n \times 2 n$ unitary matrix

$$
E_{1}=\left[\begin{array}{cc}
\tilde{U}, & 0 \\
0, & \tilde{U}
\end{array}\right], \text { then } E_{1}^{-1} J E_{1}=M_{1}(\lambda),
$$

$M_{1}(\lambda)$ defined in (6.30). Moreover, we may uniquely construct an invertible analytic matrix $E_{2}=E_{2}(\lambda)$, so that

$$
E_{2}^{-1} M_{1}(\lambda) E_{2}=\left[\begin{array}{cc}
\Lambda, & 0 \\
0, & -\Lambda
\end{array}\right], \quad \Lambda \text { as in (6.20), }
$$

with $E_{2}$ normalized in the following way: Pick a $\lambda^{\prime}$ and fix it, defining $\lambda=\varepsilon^{-1} \lambda^{\prime}$, but unlike the situation in Lemma 11.3, we shall think of $\varepsilon \rightarrow 0$. By $(6.31),(6.33)$, we have

$$
\begin{aligned}
\varepsilon B M_{1}(\lambda) B^{-1} & =M_{\varepsilon}\left(\lambda^{\prime}\right)=M_{0}\left(\lambda^{\prime}\right)+O(\varepsilon) \\
& =D \underline{\Lambda}^{\prime} D^{-1}, \text { with } \underline{\Lambda}^{\prime}=\left[\begin{array}{cc}
\Lambda^{\prime}, & 0 \\
0, & -\Lambda^{\prime}
\end{array}\right], \quad \Lambda^{\prime}=-\frac{1}{2} \lambda^{\prime},
\end{aligned}
$$

with $O$ depending on $\lambda^{\prime}$, and where we have employed Lemma 11.3 to conclude $\Lambda^{\prime}=-\frac{1}{2} \lambda^{\prime}$.

From this we infer

$$
\left(B^{-1} D\right)^{-1} M_{1}(\lambda)\left(B^{-1} D\right)=\underline{\Lambda}, \quad \Lambda=\frac{1}{2} \lambda,
$$

where by (6.34), and $B=\left[\begin{array}{cc}I, & 0 \\ 0, & \varepsilon I\end{array}\right]$, we conclude

$$
\begin{aligned}
B^{-1} D & =\left[\begin{array}{cc}
I, & 0 \\
0, & \varepsilon^{-1} I
\end{array}\right] \cdot\left\{\left[\begin{array}{cc}
I . ., & I \\
-\frac{1}{2} \lambda^{\prime}, & \frac{1}{2} \lambda^{\prime}
\end{array}\right]+O(\varepsilon)\right\} \\
& =\left[\begin{array}{cc}
I, & I \\
-\frac{1}{2} \lambda, & \frac{1}{2} \lambda
\end{array}\right]+\left[\begin{array}{cc}
O(\varepsilon), & O(\varepsilon) \\
\varepsilon^{-1} O(\varepsilon), & \varepsilon^{-1} O(\varepsilon)
\end{array}\right],
\end{aligned}
$$


$O$ depending on $\lambda^{\prime}$, and similarly from (6.34) we find

$$
\left(B^{-1} D\right)^{-1}=D^{-1} B=\left[\begin{array}{rr}
\frac{1}{2} I, & -\lambda^{-1} \\
\frac{1}{2} I, & \lambda^{-1}
\end{array}\right]+\left[\begin{array}{ll}
O(\varepsilon), & \varepsilon O(\varepsilon) \\
O(\varepsilon), & \varepsilon O(\varepsilon)
\end{array}\right] .
$$

The above estimates allow us to normalize $E_{2}$, so that, again keeping $\lambda^{\prime}$ fixed, and thus fixing the behavior of $O(\varepsilon)$ as $\varepsilon \rightarrow 0$,

$$
\left[\begin{array}{cc}
I, & 0 \\
0, & \varepsilon I
\end{array}\right] \cdot\left\{E_{2}-\left[\begin{array}{cc}
I, & I \\
-\frac{1}{2} \lambda, & \frac{1}{2} \lambda
\end{array}\right]\right\} \rightarrow 0 \text { as } \varepsilon \rightarrow 0 \text { as } O(\varepsilon),
$$

or equivalently

$$
\left\{E_{2}^{-1}-\left[\begin{array}{ll}
\frac{1}{2} I, & \lambda^{-1} \\
\frac{1}{2} I, & \lambda^{-1}
\end{array}\right]\right\}\left[\begin{array}{cc}
I, & 0 \\
0, & \varepsilon^{-1} I
\end{array}\right] \rightarrow 0 \text { as } \varepsilon \rightarrow 0 \text { as } O(\varepsilon) .
$$

We thus have constructed the invertible meromorphic matric function $E(x, y)=E_{1}(x, y) \cdot E_{2}(\lambda)$, and so we have from (6.28), the time dependence of the $H_{\alpha}$ flow (6.1) given by

$$
\left(\begin{array}{l}
p \\
\dot{p}
\end{array}\right)=P=E \cdot\left\{\exp \left[\left(E^{-1} J_{0} E\right) t\right]\right\} \cdot\left(E^{-1} P_{0}\right),
$$

(with $E$ depending on the initial data),

$$
\begin{aligned}
& =E e^{\Lambda t}\left(E^{-1} P_{0}\right), \quad P_{0}=\left[\begin{array}{c}
I \\
-\frac{1}{2} Z_{0}^{-}
\end{array}\right], \text {by }(6.44),(6.27), \\
& =\left[\begin{array}{ll}
e_{11}, & e_{12} \\
e_{21}, & e_{22}
\end{array}\right]\left[\begin{array}{cc}
\Lambda t & 0 \\
0, & e^{-\Lambda t}
\end{array}\right]\left[\begin{array}{l}
p_{1} \\
p_{2}
\end{array}\right], \quad\left[\begin{array}{l}
p_{1} \\
p_{2}
\end{array}\right]=E^{-1} P_{0}, \text { i.e., } \\
p & =e_{11} e^{\Lambda t} p_{1}+e_{12} e^{-\Lambda t} p_{2}
\end{aligned}
$$

The invertibility and meromorphicity of $E$ implies that $e_{i j}, p_{1}, p_{2}$ are meromorphic functions of the initial data. By arguments similar to the ones given above, and in Lemma 11.3, we can easily show that $e_{11}, p_{1}$ are invertible on some open set in $(x, y)$ space, and similarly for $e_{12}, p_{2}$. We thus conclude the proof of $(6.23)$, and therefore of Lemma 11.2, by proving the following statement:

Corollary 11.4. Under the hypothesis of Theorem 11, we have

$$
\begin{aligned}
& \text { (a) } \sum_{i=1}^{n} \beta_{i}^{+}=\sum_{i=1}^{n} x_{i}(0)-2 \log \left(\operatorname{det}\left(e_{11} p_{1}\right)\right), \\
& \text { (b) } \sum_{i=1}^{n} \beta_{i}^{-}=\sum_{i=1}^{n} x_{i}(0)-2 \log \left(\operatorname{det}\left(e_{12} p_{2}\right)\right)
\end{aligned}
$$

with $e_{11}, e_{12}, p_{1}, p_{2}$ as in (6.48), $\beta_{i}^{+}, \beta_{i}^{-}$as in (6.7).

Remark. This easily implies $e_{i j}^{-1}$ exists for $i, j=1,2$, by the meromorphicity of $e_{i j}, p_{1}, p_{2}$, and of $\beta_{i}^{ \pm}$, in initial data. 
Proof of Corollary 11.4. We shall just Prove (a), (b) follows similarly. Clearly it suffices to establish (6.49a) in some open set where $e_{11} p_{1}$ is nonsingular, and meromorphically continue. Since $\dot{p} p^{-1}=-\frac{1}{2} r^{-}, p(0)=I$, we compute, using (6.3),

$$
d \log (\operatorname{det} p) / d t=-\frac{1}{2} \operatorname{tr}\left(r^{-}\right)=-\frac{1}{2} \operatorname{tr} R^{-}=-\frac{1}{2} \sum_{i=1}^{n} y_{i}=-\frac{1}{2} \sum_{i=1}^{n} \dot{x}_{i}
$$

Hence $\log \operatorname{det} p+\frac{1}{2} \sum x_{i}=$ constant, and thus

$$
\begin{aligned}
\frac{1}{2} \sum x_{i}(0)= & \lim _{t \rightarrow \infty}\left(\log (\operatorname{det} p)+\frac{1}{2} \sum x_{i}\right) \\
= & \lim _{t \rightarrow \infty}\left\{\log \left(\operatorname{det}\left(e_{11} p_{1}\right)\right)+\log \left(\prod_{i=1}^{n} e^{-\lambda_{i} t / 2}\right.\right. \\
& +\left(\frac{1}{2} \sum \lambda_{i} t+\frac{1}{2} \sum \beta_{i}^{+}+O\left(t^{-1}\right)\right\},
\end{aligned}
$$

where we have used (6.48), (6.7), from which we conclude

$$
\frac{1}{2} \sum x_{i}(0)=\log \left(\operatorname{det}\left(e_{11} p_{1}\right)\right)+\frac{1}{2} \sum \beta_{i}^{+},
$$

and the corollary is proven.

I wish to thank Professor J. Moser for his many suggestions in both formulation and technical specifics, and last but not least for his enthusiasm and encouragement.

\section{References}

1. Calogero,F., Marchioro,C.: Exact solution of a one-dimensional three-body scattering problem with two-body and/or three-body inverse-square potential. J. Math. Phys. 15, 1425 -1430 (1974)

2. Sutherland, B.: Exact results for a quantum many-body problem in one dimension, II. Phys. Rev. A 5, 1372-1376 (1972)

3. Moser,J.: Three integrable Hamiltonian systems connected with isospectral deformations. Advanc. Math. 16, 1-23 (1975)

4. Lax,P.D.: Integrals of nonlinear equations of evolution and solitary waves. Comm. Pure Appl. Math. 21, 467-490 (1968)

5. Flaschka,H.: The Toda lattice, I. Phys. Rev. B 9, 1924-1925 (1974)

6. Arnold,V.I., Avez, A.: Ergodic problems of classical mechanics. Benjamin; New York 1968 (see Appendix)

7. Gallavotti,G., Marchioro, C.: On the calculation of an integral. J. Math. Anal. Appl. 44, 661-675 (1973)

8. Weinstein, A.: Normal modes for nonlinear Hamiltonian systems. Inventiones Math. 20, 47-57 (1973)

9. Sawada,K., Kotera,T.: Integrability and a solution for one dimensional $n$-particle systems with inversely quadratic pair potentials. Preprint, Tokyo University of Ed. TUETP-75-10 (1975)

10. Calogero,F.: Solution of the One-dimensional $n$-body problem with quadratic and/or inversely quadratic pair potentials. J. Math. Phys. 12, 419-436 (1971) (esp. p. 427)

11. Olshanetzky,M.A., Perelomov, A. M.: Explicit solution of the Calogero model in the classical case and geodesic flows on symmetric spaces of zero curvature. Lett. Nuovo Cimento 16, 333 (1976)

12. Adler, M.: A new integrable system and a conjecture by Calogero. November (1975) (unpublished)

13. Adler, M.: Some finite dimensional integrable systems. Preprint 6176. To appear in Proceeding of Conference on Theory and Application of Solitons, held January 1976

14. Adler,M.: Some finite dimensional integrable systems. Doctoral dissertation. Courant Institute (1976)

15. Moser,J.: The scattering problem for some particle systems on the line. To appear in Proceedings of the Conference on Dynamical Systems, held in Rio de Janeiro, summer 1976 
16. Calogero,F., Marchioro,C., Ragnisco,O.: Exact solution of the classical and quantal onedimensional many-body problems with the two-body potential $V_{d}(x)=g^{2} a^{2}\left[\sinh ^{-2} a x\right]$. Lett. Nuovo Cimento 13, 383 (1975)

17. Olshanetzky,M. A., Perelomov, A.M.: Completely integrable Hamiltonian systems connected with semisimple Lie algebras. Inventiones math. 37, 93-108 (1976)

18. Olshanetzky,M.A., Perelomov, A.M.: Completely integrable classical systems connected with semisimple Lie algebras, II. Moscow Institute of Theoretical and Experimental Physics, ITEP-27 (1976)

Communicated by J. Moser

Received February 15, 1977

Note added in proof: In a recent preprint, Kazhdan, Kostant and Sternberg have shown how to relate the Cases A, B, for $\alpha=0$ to symplectien actions of the unitary group. 